\title{
Zur Lehre von den Augenmuskellähmungen und den Störungen der Pupillenbewegung.
}

Eine vergleichende und pathologisch-anatomische, experimentelle und klinische Studie über die Augenmuskelkerne, das Ganglion ciliare, die Reflexbahnen und das Reflexcentrum der Pupille.

I. Hälfte.

Von

Dr. Ludwig Bach,

Privatdocenten und I. Assistenten der Universitäts-Augenklinik in Würzburg.

Hierzu Taf. XIII-XVI, Fig. 1-14.

Der Beginn der vorliegenden Untersuchungen des Verfassers reicht in das Jahr 1892 zurück. In diesem Jahre hatte der Verfasser Gelegenheit drei Fälle angeborener Augenmuskellähmungen genauer zu untersuchen ${ }^{1}$ ), ein Fall konnte auch pathologisch-anatomisch untersucht werden. Die Durchsicht der Serie durch das Oculomotoriuskerngebiet dieses Falles, die Durchsicht einiger anderer Serien des Oculomotoriuskernes, welche Herr Prof. C. Rieger gütigst zur Verfügung stellte, die Durchsicht ferner der vorliegenden Literatur liess in dem Verfasser den Wunsch entstehen, die Lehre von den Augenmuskellähmungen von verschiedenen Gesichtspunkten aus von Neuem zu bearbeiten. Bei der Ausführung meines Planes, besonders bei dem experimentellen Theil begegnete ich grossen, nicht geahnten Schwierigkeiten, die oft die Lust an der Arbeit erheblich sinken liessen und so kam es, dass ich erst jetzt

1) Centralblatt f. Nervenheilk. u. Psychiatrie. Februar 1893. 
nach mehreren Jahren $\mathrm{zu}$ einem gewissen, befriedigenden Abschluss gelangte.

Die vorliegende Abhandlung besteht aus einem vergleichend-anatomischen, einem pathologisch-anatomischen, einem experimentellen, und einem klinisch-statistischen $\mathrm{Ab}$ schnitt.

\section{Vergleichend anatomischer Theil.}

Den Ausführungen dieses Abschnittes liegt eine Reihe von Serien durch das Kerngebiet der Augenmuskeln beim Kaninchen, bei der Katze, beim Affen, beim erwachsenen Menschen und bei menschlichen Embryonen zu Grunde.

Als Untersuchungsmethoden wurden die Weigert'sche, Pal'sche und die Golgi-Cajal'sche Methode benutzt. Bezüglich der Technik der letzteren verweise ich auf $v$. Lenhossek"s Buch: „Der feinere Bau des Nervensystemes im Lichte neuester Forschungen". 2. Anflage. Berlin. Fischer's medicin. Buchhandlung. H. Kornfeld. 1895.

Hinsichtlich der Schnittrichtung sowohl dieser Serien als auch der im experimentellen Theil besprochenen Serien des Octlomotoriuskernes sei bemerkt, dass die Austrittsstelle des Oculomotorius am Hirnstiel als Richtungsebene benutzt wurde. Diese schräg-frontale Schnittführung hat neben anderen Vortheilen in Bezug auf die Gruppirung des Nervenkernes vor Allem den, dass man den Verlauf der Wurzelfasern besser verfolgen kann. (Siehe anch Bernheimer: Das Wurzelgebiet des Oculomotorius beim Menschen. Wiesbaden, J. F. Bergmann's Verlag 1894.)

\section{Frontalschnittserie durch das Trochlearis- und \\ Oculomotoriuskerngebiet eines Kaninchens.} Hierzu Fig. 1-3 auf Taf. XIII.

Die der Beschreibung zu Grunde liegende lïckenlose, nach Weigert behandelte Serie durch das Trochlearis- und Oculonotoriuskerngebiet eines $\mathrm{kleinen}$ Kaninchens führt in der fortlaufenden Serie durch das Gehirn die Nummern 78--136. -- 14 Schnitte kommen auf das Trochleariskerngebiet, die übrigen auf das des Oculomotorius. Die Schnitte sind durebschnittlich $35-40 \mu$ stark.

Das Kerngebiet des Trochlearis zeigt eine im Grossen und Ganzen rundliche Form und besteht der Mehrzahl nach ans ziemlich grossen Zellen von motorischem Typus. Der Trochleariskern liegt der dorsalen Seite des hinteren Längsbündels direct an. Das hintere Längsbündel ist an dieser Stelle etwas ausgehöhlt. Die Ganglienzellen schieben sich zum Theil etwas zwischen die stark markhaltigen, quer getroffenen Fasern des hinteren dorsalen Längsbündels herein. Einzelne Fasem des dorsalen Längsbündels sind etwas schräg getroffen und bedecken einen Theil der Ganglienzellen des Kernes. Zwischen den Ganglienzellen selbst sehen wir kreuz und quer ver- 
laufende markhaltige und marklose Fasern. Die Ganglienzellen sind umsponnen ron solchen Fasern. In dieser Beziehung bestehen ganz dieselben Verhältnisse im Oculomotoriuskern; dieselben sind in den Figuren 1 u. 2 auf Taf. XIII naturgetrea dargestellt. Zwischen den Querschnitten des hinteren Längsbündels tritt eine Anzahl von Fasern, welche sich zum Theil schon im Kerngebiet zu Bündeln vereinigt haben aus, ein stärkeres Bündel verläuft dorsolateral. - Das Kerngebiet ragt bis an die mediale Grenze der Längsbündelquerschnitte heran. - Der Zwischenraum zwischen der medialen Begrenzung des hinteren Langsbündels der rechten und linken Seite sowie der etwas grössere Zwischenraum zwischen den Trochleariskernen wird von einer geringen Anzahl feiner Fasem belebt. Veber deren Herkunft, sowie über den in der Regel nicht weit zu verfolgenden Verlauf kann nichts Bestimmtes ausgesagt werden. Abgesehen von diesen feinen Fasern bemerken wir an genannten Stellen auch eine Anzahl verschieden gestalteter, kleinerer Ganglienzellen, es sind dies dieselben Ganglienzellen, welche wir später anch im Zwisehenraum zwischen dem linken und rechten Oculomotoriuskern, besonders dicht gehäuft im dorsoproximalen Theile antreffen.

Das Oculomotoriuskerngebiet schliesst sich proximal direct an. das Trochleariskerngebiet an, es besteht in der Regel nur ein ganz schmaler, zellarmer Zwischenraum. - Die Form des Oculomotoriuskernes weicht zunächst nicht wesentlich von der des Trochlearis $a b$, die Lage zum hinteren Längsbündel bleibt dieselbe, die Ausdehnung ist gleich im Anfang eine etwas grössere.

An meisten fällt beim Beginn des Oculomotoriuskerngebietes das Auftreten einer grösseren Anzahl markhaltiger, bogenförmig oder

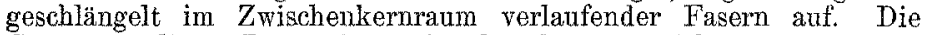
Concavität dieser Bogen ist meist dorsalwärts gerichtet. Im Beginn des Oculomotoriuskernes finden sich diese bogenartig verlaufenden Fasern hauptsächlich im dorsalen Bereich des Zwischenkerngebietes. Bald jedoch sieht man die Schlingen auch weiter herunterragen bis fast in gleiche Höhe mit der dorsalen Seite der hinteren Längsbündelquerschnitte. Man kann hier zwischen längeren und kürzeren bogenförmig verlaufenden Fasern unterscheiden. - Das Aussehen der Ganglienzellen, ihre Begrenzung durch ein Gewebe dicht verfilzter Fasern bleibt im Oculomotoriuskem ganz gleich. Einzelne grössere Ganglienzellen finden sich im distalen Abschnitt auch in dem Zwischenkernraum.

Schnitt 89. Die einzelnen Nervenfasem sammeln sich auch hier zum grossen Theil schon im Kerngebiet zu verschieden starken Bündeln, welche einen geschlungenen Verlauf zwischen den Ganglienzellen hindurch nehmen und zwischen den Längsbündelquerschnitten hindurchtreten. - Der Zwischenraum zwischen der medialen Begrenzung der Längsbündelquerschnitte ist zu Beginn des Oculomotoriuskernes nur sehr wenig von dorsoventral in gerader Richtung ziehenden Fasern bevölkert. Letztere Fasem sind ungekreuzte, wälhrend die oben beschriebenen, von dem einen Kern zum anderen ziehenden Fasern gekreuzte Fasern sind. - Zwischen den Längsbündelquerschnitten sehen wir einzelne Ganglienzellen.

Schon nach wenigen Schnitten durch den distalen Kernabschnitt ändert sich etwas die Form des Kernes, er wird mehr oval; die Spitze des Ovals liegt ventral gegen die Medianlinie zu gerichtet. 
Die dorsalen Kernabschnitte divergiren etwas. Diese Form des Kerngebietes bleibt durch eine grössere Anzahl von Schnitten erhalten, seine Böhe und Breite nimmt stetig zu.

Schnitt 96. Man gewinnt hier den Eindruck, das Kerngebiet zerfalle in eine Anzahl Unterabtheilungen. Diese Gliederming wird durch dickere Nervenfaserbündel hervorgebracht. Beim Durchsehen der Serie kann man sich nun leicht überzengen, dass diese Gliederang keine constante ist, dass sie bei ein und derselben Serie eine rasch wechselnde ist, dass sie bei der Durchsicht mehrerer Serien nicht immer in gleicher Weise auftritt. Bei Anwendung stärkerer Vergrösserungen sieht man auch, dass zwischen und unter den die Gliederung scheinbar hervorrufenden Nervenfaserbündeln ebenfalls Ganglienzelien sich finden. - Im Zwischenkernraum sehen wir nur mehr spärlich grosse Ganglienzellen.

Schnitt 102. Form und Ausdehnung des Kerngebietes sind ziemlich gleich. Gekreuzte Fasern sind noch vorhanden, die Zahl der ungekreuzten hat wesentlich zugenommen. Zwischen, ja zum Theil ausserhalb der Längsbündelquerschnitte befindet sich eíne grössere Anzahl von Ganglienzellen.

Schnitt 110. Im dorsalen Kermabschnitt sieht man noch in der Mittellinie in flachen Bogen verlanfende, sich kreuzende Faserm, die Zahl der ungekreuzten Fasern ist im Uebergewicht - An diesem Schnitt ist sehr schön der Verlauf der zu Bündeln vereinigten Oculomotoriusfasern zwischen dem Kerngebiet und dem Austritt des Nerven an der Hirnbasis zu verfolgen. Wir constatiren, dass die pinselartig das Kerngebiet verlassenden Bündel zuerst etwas nach der Medianlinie hinziehen in ganz leicht lateralwärts concavem Bogen; bald jedoch ändert sich diese Verlaufsrichtung, die Bündel wenden sich etwas lateralwärts mit geringer Convexität gegen die laterale Seite gerichtet. Bis zur Sammlung der einzelnen Bündel zum Oculomotoriusstamme wechselt die Verlaufsrichtung gerade noch einmal in der eben beschriebenen Weise.

An diesem Schnitt (vgl, auch die Fig. 2 n. 3, Taf. XIII) fällt ferner eine stärkere Ansammlung dichtgehäufter kleinerer Zellen im dorsalen Abschnitt des $Z$ wischenkernraumes auf. Wir erkennen in denselben die sogen. Edinger ${ }^{1}$ - Westphal'schen ${ }^{2}$ ) kleinzelligen Mediankerne (Bexnheimer" $\mathrm{s}^{3}$ ) paarige Mediankerne), von denen später noch ausführlich die Rede sein wird. Dieselben sind nicht scharf yon den seitlichen Hauptkernen getrennt, auch in der Mitte besteht keine scharfe Trennung. Oben sind sie breiter und divergiren etwas, unten sind sie leicht zugespitzt. Ihre Länge beträgt ungefähr 1/4 der seitlichen Hauptkeme. - In dem darunter liegenden Zwischenkernraum sind ebenfalls diese kleineren Ganglienzellen in spärlicher Anzahl vorbanden. Bemerkt sei, dass sich diese kleinen Zellen im ganzen Zwischenkernraum des Trochlearis und Oculomotorius finden, dass sie jedoch so dicht gehäuft und stark hervortretend sich nur im proxi-

1) Arch. f. Psychiatrie. Bd. XVI.

2) Arch. f. Psychiatrie. Bd. XVIII u. Neurol. Centralbl. 1888.

3) Das Wurzelgebiet des Oculomotorius beim Menschen. Wiesbaden, J. F. Bergmann's Verlag. 1894. 
malen Kernabschnitt, wo das dorsale Ende des Kemes divergirt, vorfinden.

Schnitt 117. Die Ausdehnung des Kernes hat schon wesentlich abgenommen, er hat hier mehr die Form eines Dreieckes mit ventraler Spitze. Die mediale Begrenzung des Kernes verläuft ziemlich parallel zur Medianlinie. Wir sehen hier keine sich kreuzenden Fasern mehr. Die den Kern verlassenden stäkeren Faserbündel treten mehr durch die mittleren und ventralen Längsbündelquerschnitte hindurch, eine sehr grosse Anzahl von Fasern tritt in dem Zwischenraum zwischen dem medialen Ende der Längsbündelquerschnitte aus.

Bezüglich der dorso-medialen kleinzelligen Anhäufung gilt das Gleiche, was bei der Besprechung des voraufgehenden Schnittes gesagt wurde. Von Perlia's ) Centralkern (Bernheimer's ${ }^{2}$ ) unpaarem Mediankern) ist auch hier und in den nächsten Schnitten nichts zu sehen.

Schnitt 123. Der Kern ist wieder etwas kleiner geworden und hat mehr Mandelform angenommen, das dorsale Ende divergirt etwas mehr. Die kleinzellige Anhäufung dorsomedial ist etwas weniger dicht gehäuft. Unterabtheilungen in den seitlichen Hauptkernen sind auch hier nicht vorhanden. Gekrenzte Fasern sind keine mehr vorhanden. Ueber den Faserverlauf siehe die Abbildung des nächstfolgenden Schnittes, Fig. 3 auf Taf. XIII.

Sohnitt 133. Der Kern ist bedeatend kleiner geworden, die Form ist annähernd die gleiche.

\section{Kurze Zusammenfassung der Hauptpunkte.}

Der Trochleariskern hat beim Kaninchen eine rundliche Form. Er besteht der Mehrzahl der Zellen nach aus ziemlich grossen Ganglienzellen von motorischem Typus und liegt in einer flachen dorsal gelegenen Aushöhlung der Längsbündelquerschnitte. Der Zwischenraum zwischen den beiden Trochleariskernen ist etwas grösser als der zwischen beiden Oculomotoriuskernen.

Proximal schliesst direct der Oculomotoriuskern an. Es besteht in der Regel eine mehr minder breite, zellarme Zone zwischen beiden Kernen. Der Beginn des Oculomotorius ist sofort erkennbar an dem Auftreten einer grösseren Anzahl von geschlängelt und bogenförmig verlaufenden Fasern im Zwischenkernraum, auch finden sich

1) v. Graefe's Arch. f. Ophthalm. Bd, XXXV. 4. S. 287.

2) Loc. cit. 
beim Beginn des Oculomotorius einige grössere Ganglienzellen im $Z$ wischenkernraum, während vorher nur kleinere Ganglienzellen daselbst zu finden waren. Die Ausdehnung des Kerngebietes ist anfänglich kaum grösser als die des Trochlearis, die Form ist mehr die eines Ovales, gegen die Mitte zu nimmt der Kern an Ausdehnung zu, gleichzeitig rücken die Kerne etwas mehr zusammen, im proximalen Drittel nimmt der Kern allmählich an Ausdehnung wieder ab. - Bezüglich der Form siehe man die Abbild. 1-3 auf Taf. XIII. - Im proximalen und zum Thèil im mittleren Kernabschnitt ist dorsomedial eine dichte birnförmige Anhäufung von kleineren Ganglienzellen zu sehen. Dieselben confluiren in der Medianlinie und sind auch gegen die Hauptkernmasse nicht scharf abgetrennt. Diese Anhäufung dürfte wohl der beim Menschen viel deutlicher hervortretende Edinger $\left.{ }^{1}\right)$-Westphal'sche ${ }^{2}$ kleinzellige Mediankern (Bernheimer's ${ }^{3}$ ) paarige Mediankerne) sein. Dorsal überragt dieselbe die seitlichen Kerne etwas. Thre grösste Ausdehnung ist ungefähr gleich ein Drittel der seitlichen Hauptkerne. Nach oben besteht eine geringe Divergenz. Oben ist sie breiter als unten. - Die gleichen kleinen Ganglienzellen finden sich in der Mitte und im ventralen Abschnitt des Zwischenkernraumes ebenfalls, aber weniger zahlreich. Jedoch auch im distalen Abschnitt des Zwischenkernraumes und auch zwischen den Trochleariskernen finden wir solche Zellen in unterschiedlicher Zahl. - Ein sogenannter unpaarer, grosszelliger Mediankern nach Bernheimer ${ }^{4}$ ) (Perlia's ${ }^{5}$ ) Centralkern) ist nicht vorhanden.

1) Arch. f. Psychiatrie. Bd. XVI.

2) Arch. f. Psychiatrie. Bd. XVIII u. Neurol. Centralbl. 1888.

3) Das Wurzelgebiet des Oculomotorius beim Menschen. J. F. Bergmann's Verlag. Wiesbaden 1894.

4) Ibidem.

5) v. Graefe's Arch. f. Ophthalm. Bd. XXXV. 4. S. 287. 
Zwischen den Längsbündelquerschnitten, ja auch ausserhalb derselben finden sich besonders distal und in der Mitte Ganglienzellen in unterschiedlicher Zahl von dem gleichen Typus wie die Zellen der Hauptkernmasse. Sie sind sicher Bestandtheile des Kerngebietes.

Im distalen Drittel findet sich eine grosse Mehrzahl von gekreuzten Fasern. Dieselben verlassen das Kerngebiet zwischen den seitlichen, besonders den dorsal gelegenen Längsbïndelquerschnitten. Im mittleren Drittel finden wir nur vereinzelte gekreuzte Fasern, im proximalen Drittel gar keine mehr.

Ungekreuzte Fasern sind distal sehr wenig zu sehen, im mittleren Drittel sind sie in der Mehrzahl, proximal ausschliesslich vorhanden.

Eine scharfe Gliederung in mehrere Unterabtheilungen existirt nicht.

Die Querschnitte des dorsalen hinteren Längsbündels nehmen von hinten (distal) nach vorn (proximal) allmählich an Mächtigkeit ab.

\section{Frontalschnittserie durch das Trochlearis- und \\ Oculomotoriuskerngebiet einer grossen Katze. Hierzu Fig. $4-6$ auf Taf. XIII u. XIV.}

Es liegt eine lückenlose Serie von 123 (ca. $35 \mu$ starken) Schnitten vor. 26 Schnitte betreffen das Trochlearis-, 97 das Oculomotorinskerngebiet. Die Serie beginnt distal und führt die Nummern 6-129.

Schnitt 17. Derselbe geht durch die Mitte des Trochleariskerngebietes. Die Kernmasse liegt in einer starken dorsoconcaven Aushöhlung des hinteren Längsbündels. Die Form ist nicht wie beim Kaninchen nahezu eine runde, sondern mehr oval. Der Kern ragt nicht ganz bis an die mediale Grenze der Querschnitte des hinteren Längsbündels heran. Er besteht grösstentheils aus grossen Ganglienzellen von motorischem Typus, welche in ein Maschenwerk vielfach sich verflechtender feiner Fasern eingebettet sind. Einzelne Zellen sind zwischen die Längsbiundelquerschnitte vorgeschoben. Schon innerhalb des Kerngebietes sammeln sich Fasern zu stärkeren Bündeln, welche in gewundenem Lauf durch das Kerngebiet ziehen. Zwischen den Längsbündelquerschnitten treten Faserbündel aus, die sich nur eine kurze Strecke verfolgen lassen. Ein stärkeres Bündel tritt beiderseits medial aus dem Kerngebiet aus und zieht in gerader, 
dorsoventraler Richtung weiter. - Es besteht ein breiter Zwischenraum zwischen den beiden Trochleariskernen.

Derselbe wird von einer grösseren Anzahl meist feiner Fasern belebt, welche in den verschiedensten Bichtungen verlaufen. Wir sehen Fasern, welche dorsal rom Kerngebiet herkommen und in ziemlich gerader Richtung dorsoventralwärts verlaufen, andere Fasern biegen schlingenförmig um, einzelne ziehen in gewundener Linie von einem Kerngebiet zum anderen.

Schnitt 25. Die Höhe des Kerngebietes hat etwas abgenommen, die seitliche Ausdehnung ist gleich geblieben, es zeigt demnach das Kerngebiet die Form eines Ovals. Die austretenden Faserbündel lassen sich hier etwas weiter verfolgen. In dem medialen Theil des Kerngebietes fallen einige sehr stark mit Mark bekleidete Fasern auf, welche in dem Kerngebiet $z u$ endigen scheinen. Es handelt sich hierbei wohl mit ziemlicher Sicherheit um längs und schräg getroffene Fasern des hinteren Längsbündels.

Schnitt 33. Mit diesem Schnitt sind wir in das Oculomotoriuskerngebiet eingetreten, nachdem zwei Schnitte vorausgegangen waren, welche das Grenzgebiet zwischen dem Trochlearis- und Oculomotoriuskern darstellten. In diesen beiden Schnitten gewahrten wir ein dichtes Maschenwerk von Fasern, dazwischen nur vereinzelte Ganglienzellen. Am Schnitte 33 fällt sofort auf, dass jetzt der Zwischenraum $z$ wischen dem Kerngebiet der rechten und linken Seite durch eine viel grössere Anzahl markhaltiger Faserzüge belebt ist. Diese Fasern verlaufen zum Theil in gerader Richtung dorsoventral, zum Theil nehmen sie einen geschlungenen Verlauf von einer Seite zur anderen (sich lrueuzende Fasern).

Letztere Fasern treffen wir hier ansschliesslich im dorsalen. Theil des $Z$ wischenkernraumes, ebendaselbst sehen wir eine Anzahl von grossen Ganglienzellen. - Das Kerngebiet zeigt hier eine ovale Form und liegt in einer sehr flachen Aushöhlung der dorsalen Längsbündelquerschnitte. Die Ganglienzellen zeigen ganz denselben Typus wie die des Trochleariskerngebietes und liegen ebenfalls in einem Filz feiner Fasern. - Die austretenden Faserbündel verlassen das Kerngebiet zwischen den seitlichen Längsbündelquerschnitten, aber auch bier schon in grösserer Zabl in dem Zwischenraum zwischen den medialen Querschnitten der rechten und linken Seite. Siehe Fig. 4, Taf. XIII, Schnitt 36.

Schnitt 38. Der Korn hat an Höhe und Breite ungefähr um das Doppelte zugenommen. Der Zwischenraum zwischen dem Kerne der rechten und linken Seite ist mehr mit Zellen belebt. Besonders am dorsalen Ende des Zwischenkernraumes fällt eine die Seitenkerne etwas überragende Zellanhäufung auf. Gekreuzte Fasern sind zahlreich vorhanden, besonders im dorsalen und mittleren Bereich des Zwischenkernraumes treffen wir die von denselben gebildeten, theils langeren, theils kürzeren dorsoconcaven Schlingen an. Fig. 4, Taf. XIII, Schnitt 36.

Schnitt 42. Die Form und Ausdehnung des Kerngebietes ist die gleiche geblieben. Das Bild ist wesentlich nur dadurch geändert, dass die Zellanhäufung im Zwischenkernraum dorsal geringer geworden ist. Abgesehen von dem am meisten ventral gelegenen Theil des $\mathrm{Zwischenkernrawmes}$ ist hier überall in demselben eine Anzahl von grossen Ganglienzellen (gleich denen der seitlichen Hauptkeme) vorhanden, wodurch gewissermaassen eine Brücke zwischen dem Kern- 
gebiet der rechten und linken Seite hergestellt wird. - Gekreuzte Fasern sind noch in grosser Zahl vorhanden; ihre Schlingen ragen zum Theil weiter in den ventralen Abschnitt des Zwischenkernraumes binein.

Sowohl am vorigen wie an diesem Schnitte sind nur wenige Ganglienzellen zwischen den Querschnitten des hinteren Längsbündels vorhanden.

Schnitt 50. Das Kerngebiet hat sowohl an Länge als Breite zugenommen. Es besteht nahezu gar keine Trennung mehr in ein Kerngebiet der rechten und linken Seite, da der frühere Zwischenkernraum von zahlreichen grossen und kleinen Ganglienzellen bevölkert wird; auch die Querschnitte des hinteren Längsbündels sind in der Mittellinie ventral ganz zusammengerückt. - Gekreuzte Fasem sind in grosser Anzahl vorhanden. - An diesem Schnitte erscheint die seitliche Hauptkerngruppe wieder in einzelne Unterabtheilungen getrennt. Diese Tremnung wird durch ståxkere Faserbündel bedingt. Sieht man sich in Bezug anf die Unterabtheilungen die angrenzenden Sexienschnitte an, so constatirt man hier, wie an anderen Kernabschnitten, dass in Bezug auf die Unterabtheilungen das Bild ein rasch wechselndes ist und dass durchaus keine scharfe und wirkliche Abtrennung dex einzelnen Ganglienzellengruppen existirt; auch in diesen scheinbaren Abgrenzungsbezirken liegt immer eine grössere oder geringere Anzahl yon Zellen, wovon man sich bei starker Vergrösserung leicht überzeugen kann.

Schnitt 70. Siehe Fig. 5, Taf. XIV. Das Kerngebiet hat wieder etwas an Ausdehnung zugenommen; das Zwischenkerngebiet zeigt immer noch zahlreiche grosse und kleinere Ganglienzellen. Es bildet das in der Mittellinie confluirende Kerngebiet der rechten und linken Seite ein Dreieck, dessen Spitze ventral gelegen ist. - Gekreuzte Fasern sind auch hier vorhanden und zwar im ganzen Bereich der Mittellinie.

Während in den distal gelegenen Schnitten die stärkeren, aus dem Kerngebiet austretenden Faserbündel meist zwischen den dorsal gelegenen Querschnitten des hinteren Längsbündels austraten, treten jetzt die stärkeren Faserbündel mehr zwischen den in der Mitte und ventral gelegenen Längsbündelquerschnitten hindurch.

Schnitt 89. Die Ausdehnung des Kerngebietes hat wieder um ein geringes abgenommen, die Form ist die gleiche geblieben. $A b-$ gesehen von dern am meisten ventral gelegenen Abschnitt der Mittellinie ist auch hier fast gar kein zellfreier Raum zwischen dem Kerngebiet der rechten und linken Seite vorhanden.

In dem dorsalen Abschnitt der Mittellinie sehen wir eine stärkere Anhäufung von kleineren Ganglienzellen, welche das dorsale Ende der seitlichen Hauptkerne etwas überragt. In dieser Zellanhäufung dürfen wir den kleinzelligen Mediankern Edinger ${ }^{1}$ )Westphal's'), Bernheimer's $s^{3}$ ) paarige Mediankerne erblicken. Es muss jedoch bemerkt werden, dass diese kleinzellige Anhäufung in der Mittellinie nicht seharf getrennt ist und dass auch keine irgendwie scharfe Trenmung von den seitlichen Hauptkernen vorhanden ist. Siehe Fig. 5, Taf. XIV u. Fig. 6, Taf. XIV, Schnitt 72 u. 102.
-1) Loc. cit.
2) Loc. cit.
3) Loc. cit. 
Unterhalb dieser kleinzelligen Anbäufung befindet sich in der Mittellinie eine Anzahl von Ganglienzellen, welche sich in ihrer Grösse ete. nicht von denen der seitlichen Hauptkerne unterscheiden. Man könnte in ihnen den Centralkern Peri ia's ${ }^{1}$ ) und den unpaaren Mediankern Bernheimer's ${ }^{2}$ ) erblicken, doch ist hierzu zu bemerken, dass eine scharfe Trennung dieser Zellen ron denen der Seitenhauptkerne hier nicht rorhanden ist und dass wir hier die grossen Ganglienzellen in dem $Z$ wischenkernraum in einem viel lüngeren und breiteren Raume antreffen.

In der Serie durch das Oculomotoriuskerngebiet einer anderen, etwas kleineren Katze finde ich diese in and $\mathrm{zu}$ beiden Seiten der Mittellinie vorhandene Zellanhäufung viel schärfer gegen die seitlichen Hauptkerne abgegrenzty doch kann ich anch hier keinen zellfreien Raum zwischen dem sog. unpaaren Mediankern und den seitlichen Hauptkernen finden.

Auch in diesem Schnitte sind noch gekreuzte Fasern zu sehen, zahlreicher in dem ventralen und mittleren Kernabschnitt als in dem dorsalen.

Die austretenden stärkeren Faserbündel verlassen zumeist das Kerngebiet $z$ wischen den mehr ventral gelegenen Längsbündelquerschnitten. Sie verlaufen S-förmig gekrümmt nach der Hirnbasis und zwar verlaufen die einzelnen Faserbündel eine grössere Strecke weit getrennt voneinander. Erst nahe der Hirnbasis vereinigen sich die einzelnen Faserbündel zum Stamme des Nervus oculomotorius.

Schnit 116. Die Ausdehrung des Kerngebietes hat wesentlich abgenommen. Die seitlichen Hauptkerne haben jetzt eine mehr ovale Form, die Spitze des Ovals nach unten. Dorsalwärts divergiren dieselben. Grössere Ganglienzellen sind im Zwischenkernraum nur wenige vorhanden, dahingegen ist die Anhäufung kleinerer Zellen im dorsalen Bereich des Zwischenkemraumes noch vorhanden, ja sie hat an Ausdebnung etwas zugenommen. Eine Trennung in zwei $\mathrm{Ab}$ schnitte lässt sich auch hier nicht feststellen; die Abgliederung gegen die seitlichen Hauptkerne ist etwas mehr ausgesprochen. Diese kleinzellige Anhäufung überragt die seitlichen Hauptkerne dorsalwärts etwas und ist oben etwas breiter; eine scharfe Abgrenzung besteht auch nach oben nicht, dieselben kleineren Ganglienzellen sehen wir auch in weiter Ausdehnung seitlich und dorsalwärts, nur sind dieselben etwas weniger dicht gehäuft. - Thre Ausdehnung beträgt ungeführ ein Viertel der Ausdehnung der seitlichen Hauptkeme.

Mit den nun folgenden Schnitten nimmt die Ausdehnung des Kerngebietes raseh ab; die ovale Form der seitlichen Keme bleibt bis zum Schluss erhalten.

Bis an das Ende sehen wir einzelne grössere Ganglienzellen in dem Zwischenkerngebiet. - Die dorsomediale kleinzellige Anhäufung wird gegen das proximale Ende geringer und etwas woniger dicht gehäuft, ist jedoch bis zum Schluss, ja darüber hinaus zul sehen.

Ebenso können wir bis zum Schluss in dem Zwisehenkernraum horizontal verlaufende Fasern (gekreuzte?) beobachten.

1) Loc. cit.

2) Loc. cit. 
Zusammenfassung der erhobenen Befunde.

Der Trochleariskern hat in der vorliegenden Serie einer ziemlich grossen Katze eine Länge von nicht ganz $1 \mathrm{~mm}$. Seine Form ist eine ovale. Er liegt in einer starken dorsoconcaven Aushöhlung des hinteren Längsbiundels. Zwischen dem Kerngebiet der rechten und linken Seite besteht ein ziemlich breiter $Z$ wischenraum, die Kerne erstrecken sich nicht ganz bis an den medialen Rand der Längsbündelquerschnitte. $\mathrm{Er}$ besteht aus verschieden grossen Zellen, die grossen Zellen herrschen etwas vor und finden sich anscheinend hier auch relativ in grösserer Zahl als im Oculomotoriuskern, doch sind in letzterem sicherlich eine grosse Anzahl gleich grosser Zellen vorhanden. - Vereinzelte Zellen liegen $\mathrm{zwischen} \mathrm{den} \mathrm{Längsbündelquerschnitten.}$

Das Oculomotoriuskerngebiet, Fig. 4, 5 u. 6 auf Taf. XIII u. XIV, hat in der vorliegenden lückenlosen Serie eine Ausdehnung von etwas über $3 \mathrm{~mm}$. Die Trennung zwischen Trochlearis- und Oculomotoriuskern ist deutlich ausgesprochen, jedoch besteht keine breitere zellfreie Zone zwischen beiden Kerngebieten. Der Beginn des Oculomotoriuskernes wird sofort an dem Auftreten einer grösseren Anzahl markhaltiger Fasern in dem $Z$ wischenraum zwischen beiden. Kernen kenntlich. Diese Fasern verlaufen zum Theil in gerader Richtung dorsoventral, zum Theil nehmen sie einen gewundenen Lauf, bilden dorsoconcave Schlingen in dem Zwischenkernraum. Fasern mit letzterem Verlauf treffen wir beim Beginn des Oculomotoriuskernes ausschliesslich zwischen dem dorsalen und mittleren Kernabschnitt. Es sind Fasern, welche sich kreuzen, Fasern, welche von Zellen des Kernes der einen Seite entspringen, in der beschriebenen Weise zum anderen Kerne hinüberziehen, zwischen dessen Zellen, zum Theil bereits zu Bündeln gesammelt, sich in gewundenem Verlauf hindurchschlängeln und zwischen den Längsbündelquerschnitten, vornehmlich dorsal, austreten. 
Neben diesen Fasern fällt aber auch sofort beim Beginn des Oculomotorius auf, dass in dem dorsalen Bereich des Zwischenraumes zwischen Kern der rechten und linken Seite sich eine grössere Anzahl ron Ganglienzellen, gleich denen der seitlichen Hauptkernmasse findet. Neben diesen grossen Ganglienzellen fiuden sich auch kleinere von verschiedener Form und Grösse, letztere finden sich aber auch im ventralen Abschnitt des Zwischenkernraumes, fanden sich auch schon zwischen den Trochleariskernen. Ueber die Form, Grösse, Zahl etc. der Zellen kann man sich natürlich viel besser an Präparaten orientiren, welche nicht nach Weigert behandelt, sondern mit Thionin, Methylenblau oder Toluidinblau gefärbt wurden.

Die Längsbündelquerschnitte besitzen distal eine grosse Mächtigkeit, proximal nehmen sie etwas an Ausdehnung ab.

Der Kern nimmt sehr rasch nach vorn an Höhe und auch etwas an Breite zu und erhält sich dann auf eine grössere Strecke bei annähernd gleicher Ausdehnung. Sehr auffällig ist bei der Katze, dass bald der ganze $Z$ wischenkernraum von Zellen gleich denen der seitlichen Hauptkernmasse belebt wird und es nahezu in ganzer Ausdehnung des Kerngebietes bleibt, es fliesst gewissermaassen die Kernmasse der linken und rechten Seite in der Mittellinie zusammen zu einem grossen nahezu dreieckigen Kerngebiet.

Sehr bald gewahren wir auch, dass die immer schon in dem Zwischenkernraum vorhandenen kleineren Ganglienzellen anfangen, im dorsalen Bereich etwas dichter gehäuft zu liegen. Fs sieht die betreffende Parthie etwas heller gefärbt im Schnitt aus, sie überragt nach oben die seitliche Hauptkernmasse. Auf den beigegebenen Abbildungen, die vollständig naturgetreu sind, kommt aber gut zum Ausdruck, dass ganz genau dieselben Zellen sich auch seitlich, allerdings weniger dicht gehäuft und auch nach oben befinden. 
Bei der beschriebenen Serie finden sich auffällig wenig Zellen zwischen den Längsbündelquerschnitten; etwas mehr fand ich bei einer anderen Serie, jedoch immer noch relativ wenig im Vergleich zum Kaninchen, Affen und Menschen.

Eine durchgreifende Trennung der seitlichen Hauptkernmasse in eine Anzahl Unterabtheilungen, eine wirkliche Gliederung ist nicht vorhanden, wie man sich bei der Durchsicht von Serien leicht iiberzeugen kann.

In dem proximalen Drittel des Kerngebietes divergiren die dorsalen Kernabschnitte etwas und in den dadurch entstehenden $Z$ wischenraum zwischen beiden Kerngebieten rückt die oben bereits erwähnte kleinzellige, dicht gehäufte und deshalb (?) wohl heller aussehende Ganglienzellengruppe herein. Wir erkennen in derselben den sogenannten Edinger ${ }^{1}$ - Westphal'schen ${ }^{2}$ ) Kleinzelligen Mediankern (Bernheimer's") ,paarige Mediankerne"). Zu bemerken ist hierbei, dass keine scharfe Trennung dieser Zellanhäufung in der Medianlinie besteht und dass auch die Abtrennung von der seitlichen Hauptkernmasse nur wenig scharf ist. Es sind diese Verhältnisse zu betonen gegenuiber den von anderer Seite gemachten Angaben. Zu betonen ist hier auch, dass wir ganz dieselben Zellen etwas weniger dicht gehäuft dorsal vom Kerngebiet in weiter Ausdehnung finden, dass wir ganz dieselben Zellen in der ganzen Ausdehnung des Zwischenkernraumes und darüber hinaus - sowohl distal als proximal - finden.

Von einem "Centralkern" Perlia's ${ }^{4}$ ) oder einem ,unpaaren Mediankern" Bernheimer's s) kann man bei dieser Serie nicht sprechen, denn einmal sind die im sogenannten Zwischenkernraum vorhandenen Zellen nicht abgegrenzt, gegenüber den seitlichen Hauptkernen und dann finden sie sich auch über ein viel grösseres Areal. - Bemerkt sei,
1) Loc, cit.
2) Loe, eit.
8) Loc. cit.
4) Loc. cit. 5) Loc. cit. 
dass ich bei einer anderen Serie eine schärfere Abgrenzung - aber auch keine vollständige - dieser im Zwischenkernraum gelegenen grossen Ganglienzellen fand.

Im proximalen Drittel nimmt die Ausdehnung des Kerngebietes rasch $a b$.

Wir finden auch bei der Katze gekreuzte und ungekreuzte Fasern. Die ungekrenzten sind anscheinend in der ganzen Ausdehnung? des Kerngebietes vorhanden, die gekreuzten finden sich vornehmlich distal, erhalten sich aber vereinzelt anscheinend bis fast an das proximale Fnde.

Die das Kerngebiet verlassenden Faserbündel bleiben bis fast an die Basis des Gehirnes heran isolirt, erst kurz zuvor vereinigen sie sich zum Stamme des Oculomotorius. Diese Thatsache kann wichtig werden für die Auffassung isolirter Lähmungen.

\section{Frontalschnittserie durch das Trochlearis- und Oculomotoriuskerngebiet eines Affen (Hapale Jacchus) nach Weigert. Hierzu Fig. 7-9 auf Taf. XIV u. XV.}

Das Trochlearis- und Oculomotoriuskerngebiet wurde in eine lückenlose Serie von 139 durchschnittlich $30-35 \mu$ starke Schnitte zerlegt. Die Schnitte durch das Trochleariskemgebiet führen in der Serie die Nummerm 1-32, die dureh das Oculomotoriuskerngebiet die Nummern 33-139.

Der Trochleariskern zeigt eine im Grossen Ganzen rundliche Form, er liegt in einer flachen dorsalen Aushölung der hinteren. Längsbündelquerschnitte und reicht bis an das mediale Ende derselben heran. Er besteht aus unterschiedlich grossen Ganglienzellen. von motorischem Typus. Die einzelnen Zellen liegen in einem zierlichen Fasernetzwerk. - Die austretenden Faserbuindel verlassen das Kerngebiet zwischen den Querschnitten des hinteren Längsbündels, können aber nur ganz kurz nach aussen davon verfolgt werden, worauf sie verschwinden. - An der lateralen Grenze des Kernes fällt eine reichliche Anzahl von theils quer, theils schräg getroffenen, stark markhaltigen Fasern auf; dieselben Fasern sehen wir als dorsale Begrenzung des Kernes, in geringer Zahl sind sie auch an der medialen Grenze zu sehen. - Es besteht ein ziemlich breiter Zwischenraum zwischen dem Kern der rechten und linken Seite; derselbe ist grösser als der zwischen den Oculomotoriuskernen. 
In dem Zwischenraum zwischen beiden Kernen sehen wir nur eine geringe Anzahl von feinen Fäserchen, die grösstentheils in dorsoventraler Richtung, zum Theil auch in mehr horizontaler Richtung verlaufen.

Schnitt 33. Das Bild hat sich etwas geändert, insoferm als jetzt eine grössere Anzahl von theils feinen, theils dickeren Fasern in dem dorsalen Abschnitt des Zwischenkernraumes sichtbar wird, welche sowohl in dorsoventraler als in horizontaler Richtung verlaufen. Die in horizontaler Richtung ziehenden Fasem nehmen theils einen geschlängelten Verlauf, theils bilden sie Bogen. (Siehe Fig. 7 auf Taf. XIV.) Es sind dies sich kreuzende Oculomotoriusfasern. Zellen des Oculomotoriuskernes sind an diesem Schnitte nur vereinzelte $\mathrm{zu}$ sehen, er bildet die Grenzzone zwischen Trochlearis- und Oculomotoriuskern.

In den nächsten Schnitten ändert sich insofern das Bild wesentlich, als nicht bloss eine grössere und auch weiter herabreichende Anzahl yon Fasern zwisehen beiden Kern sichtbar wird, sondern als auch eine Ansammlung von grossen Ganglienzellen in dem dorsalen Gebiet des Zwischenkernraumes sichtbar wird, welche durchaus den Typus der Zellen der Hauptkernmasse darbieten. Die Form des Oculomotoriuskemes ist z11 Beginn eine leicht ovale. (Siehe Fig. 7 auf Taf. XIV.)

Es wurde oben bei der Beschreibung eines Schnittes ungefähr durch die Mitte des Trochleariskernes der Thatsache Erwähnumg gethan, dass die dorsale Begrenzung des Kernes durch ein dickes Bündel von markhaltigen, grobcalibrigen Fasern gebildet wird, die zum Theil sich auch etwas in das Kerngebiet hineinerstrecken. Man gewann an manchen Schnitten durch den Trochleariskern den Eindruck, als ob der Trochleariskem von dem hinteren Längsbündel fast vollständig umfasst würde.

Mit dem Beginn des Oculomotorius ändert sich das Verhältniss der Kernmasse zu dem hinteren Längsbündel etwas. Wir sehen hier den Oculomotoriuskern in einer ziemlich starken dorsoconcaven Aushöhlung des hinteren Längshündels liegen. An dem oberen Ende ragt das Längsbündel etwas in die Kernmasse herein, jedoch lange nicht so stark, wie wir das beim Trochlearis sahen. - An den ersten Sehnitten durch den Oculomotoriuskern sehen wir dorsal an den Kern anschliessend ebenfalls eine Anzahl Fasern, die jedoch einen ganz anderen Typus der Anordnung darbieten. In etwas weniger dichter Anordmung sehen wir in der ganzen Länge des Oculomotoriuskernes ein Netzwerk von Fasern am dorsalen Ende.

Die aus den Kerngebiet austretenden Faserbündel verlassen dasselbe lateral, die stärkeren treten hauptsächlich zwischen den dorsal gelegenen Längsbündelquerschnitten aus. In den distalsten Schnitten sah ich keine Fasern, welche im Zwischenkernraum in gerader dorsoventraler Richtung bis zu den Längsbündelquerschnitten hin zu verfolgen waren.

In der eben beschriebenen Weise bleiben die Verhältnisse durch ungefähr 15 Schnitte hindurch.

Nun ändern sich allmäblich die Verhältnisse insofern, als die Form des Kernes etwas mehr oval wird, die Spitze des Ovals ist der ventralen Seite zugekehrt. 
Die im dorsalen Abschnitt des Zwischenkernraumes gelegenen grossen Ganglienzellen sind etwas spärlicher gevorden.

Man ist bei der Betrachtung der Schnitte dieser Gegend versucht, einzelne Unterabtheilungen des Kernes anzunehmen, da die Zellen durch theilweise ziemlich starke Faserbündel auseinandergedrängt werden. Sieht man jedoch genaner zu, so constatirt man, dass theils unter, theils über und zwischen diesen Faserbündeln ebenfalls Ganglienzellen sichtbar werden. Vergleicht man die einzelnen Kernabschnitte in einer Anzahl von Schnitten der Serie miteinander, so ergiebt sich, dass das Bild del" Gliederung ein ziemlich wechselndes ist, sich nicht durch eine grössere Anzahl von Schnitten in constanter Weise erhält. Man überzengt sich leicht, dass die durch die stärkeren Faserbündel geschaffene Gliederung des Kernes keiner wirklichen anatomischen Trennung in eine Anzahl von Unterabtheilungen entspricht, dass diese Gliederung eine scheinbare, eine rasch wechselnde ist. - Wir haben es somit beiderseits nur mit einem Hauptkern $\mathrm{zu}$ thon.

Schnitt 54. Im dorsalen Bereich des Zwischenkernranmes sehen wir eine Anzahl schlingenförmig von einem Kern zum andern verlaufender Fasern (gekreuzte Fasern), ausserdem ist hier der ganze Zwischenkernraum yon einer grossen Anzahl von dorsoventral verlaufenden Fasern belebt. - Zwischen den Querschnitten des hinteren Längsbündels treten überall Faserbündel hindurch, die stärkeren Bündel zwischen den mehr medial gelegenen Querschnitten. Dieselben können nicht sehr weit nach aussen von den Längsbündelquerschnitten verfolgt werden.

Einzelne Ganglienzellen sehen wir zwischen den Längsbündelquerschnitten, dieselben gehören sicher zu dem Oculomotoriusłern.

Schnitt 71. Siehe Fig. 8 anf Taf. XIV. Der Kem hat jetzt mehr eine Dreiecksform mit dorsal gelegener Basis und abgestumpfter ventraler Spitze. Die dorsale und mediale Begrenzung ist ziemlich scharf, die laterale Begrenzung ist eine unregelmässige in Folge der vorspringenden Längsbündelquerschnitte. $\mathrm{Er}$ ist jetzt noch einmal so gross wie in den distalsten Schnitten.

Zwischen den Längsbündelquerschnitten sind einzelne grosse Ganglienzellen zu sehen, im Zwischenkernraum sind nicht mehr wie im distalsten Bereich dorsal grosse Ganglienzellen rom Typus der seitlichen Hauptkerne vorhanden, sonderm wir sehen im ganzen Zwischenkemraum nur vereinzelte kleinere Ganglienzellen.

Die gekreuzten Fasern ragen mit ihren Schlingen zum Theil bis in die Mitte des Zwischenkernraumes herein. Die das Kerngebiet verlassenden stärkeren Faserbündel treten alle zwischen den mittleren and medialen Längsbündelquerschnitten und in dem Zwischenraum zwischen den Querschnitten der rechten und linken Seite aus. Sie können jetzt etwas weiter nach aussen rom Kerngebiet verfolgt werden.

Schnitt 84. Siehe Fig. 8 auf Taf. XIV. Der Kern ist in dorsoventraler Richtung etwas länger geworden, hat sich aber dorsal etwas verjüngt.

Der Faserverlauf im Kerngebiet und die Austrittsstellen sind gleich geblieben. Die mächtigen, zwischen den mittleren und medialen Längsbündelquersehnitten hindurehtretenden Faserbündel lassen 
sich bis zur Himbasis verfolgen. Sie verlaufen auf der ganzen Strecke vom Kern bis kurz vor der Hirmbasis getrennt und nehmen einen S-förmigen Lauf.' Sie ziehen durch den rothen Kern theils hindurch, theils medial an demselben vorbei. Erst kurz vor dem Austritt an der Basis vereinigen sich die Faserbündel zum Stamme des Oculomotorius und man kann hier schon beobachten, wie die zwischen den medial gelegenen Längsbündelquerschnitten hindurchtretenden Faserbündel schliesslich auch die mediale Parthie des Nervenstammes darstellen.

Schnitt 106. Siehe Fig. 9 auf Taf. XV. Die Ausdehnung des Kerngebietes hat wieder etwas abgenommen, sowohl der Breite als der Länge nach. Das dorsale Ende hat sich mehr verjüngt. Die beiden Kerne liegen fast ganz parallel zu einander.

An diesem Schnitt, wie auch schon an einer Anzahl der vorausgegangenen nimmt man anschliessend an das dorsomediale Ende der seitlichen Hauptkerne eine Ansammlung kleinerer Ganglienzellen wahr, welche an den sogen. ,kleinzelligen Mediankern" Edinger ")Westphal's ${ }^{2}$ ), (paarige Mediankerne Bernheimer's ${ }^{3}$ ) erinnern. Die Zellen liegen nicht so sehr dicht gehäuft, sie fliessen in der Mittellinie zusammen und sind auch von den seitlichen Hauptkernen nicht scharf abgetrennt. Dorsalwärts finden wir dieselben Zellen, weniger dicht gehäuft, in weiter Ausdehnung. Auch in dem mittleren und ventralen $Z$ wischenkernraum sind diese Zellen in geringer Zahl vorhanden.

In geringer Entfernung nach oben von dem dorsalen Ende des Kerngebietes sehen wir in der Mittellinie ūnd deren Umgebung eine kleine Anhäufung von Ganglienzollen, die etwas grösser and dunkler aussehen, wie die vorhin beschriebenen kleinen Zellen im dorsalen Bezirk des Zwischenkernraumes. Diese kleine Zellgruppe findet sich in ziemlich weiter Ausdehnung. Beziehungen zum Oculomotorius konnte ich nicht feststellen. Dieselbe Zellgruppe findet sich sehr charakteristisch auch bei der Katze, vorhanden ist sie aber auch beim Menschen und Kaninchen.

Zwischen den Längsbündelquerschnitten sehen wir besonders dorsal einzelne Ganglienzellen rom Typus der Zellen der Hauptkerne.

Gekreuzte Fasern sind hier nur mehr ganz vereinzelt zu treffen.

Zwischen den dorsalen Längsbündelquerschnitten treten nur melir spärliche Fasem aus, dahingegen verlassen mächtige Bündel das Kerngebiet in dem Zwischenraum zwischen den medialen Enden der Längsbündelquerschnitte und in deren nächster Nähe. - Die austretenden Faserbündel sind auch hier bis fast an die Hirnbasis zu verfolgen und nehmen hierbei einen S-förmigen Verlauf. Sie ziehen grösstentheils medial an dem rothen Kern vorbei. Auch. an diesem Schnitt ist leicht $z u$ ersehen, dass die mehr lateral austretenden Faserbündel den lateralen, die medial gelegenen den medialen Theil des Oculomotoriusstammes schliesslich bilden.

Schnitt 125. Siehe Fig. 9 anf Taf. XV. Die Ausdehnung des Kernes hat wieder abgenommen, er hat eine ovale Form, ist dorsal etwas schmäler als ventral. Ziemlich durch die Mitte des Kernes
1) Loc. cit.
2) Loc. cit.
2) Loc. cit, 
zieht beiderseits ein stärkeres Faserbündel, wodureh scheinbar eine Gliederung des Kernes in einen dorsolateralen und medioventralen Theil hervorgebracht wird.

Die bei dem vorigen Schnitte beschriebene kleinzellige Anhäufung an dem dorsomedialen Ende ist auch an diesem Schnitte wahrzunehmen.

Ventral davon kann man an diesem Schnitte und denen der Umgebung einzelne grössere Ganglienzellen wahrnehmen, worin man eine Andeutung des sogen. Perlia'sehen ${ }^{1}$ ) Centralkernes (Bern heime $r^{\prime}{ }^{2}{ }^{2}$ ) unpaarer Mediankern) erblicken könnte.

Ganz vereinzelte gekreuzte Fasern sind scheinbar auch hier noch vorhanden.

Bezüglich der ungekreuzten Fasern und der austretenden Faserbündel gilt das bei der Beschreibung des vorigen Schnittes Gesagte.

Die Längsbündelquerschnitte haben von hinten (distal) nach vorn (proximal) allmählich sehr an. Mächtigkeit abgenommen.

Mit den nun folgenden Schnitten nimmt das Kerngebiet rasch an Ausdehnung ab.

Kurze $Z$ usammenfassung der Hauptpunkte.

Der Trochleariskern ist von rundlicher Form und besitzt ungefähr ein Drittel der Länge des Oculomotoriuskernes. Der Zwischenraum zwischen dem Kern der rechten und linken Seite ist ein ziemlich breiter, grösser als der zwischen beiden Oculomotoriuskernen. Der Kern liegt dorsal den hinteren Längsbündelquerschnitten an, in einer flachen Aushöhlung der letzteren. Der Kem selbst besteht aus zumeist ziemlich grossen Zellen, in dem Zwischenraum zwischen beiden Kernen hingegen befinden sich nur kleinere rundliche und spindelförmige Zellen. Diese kleineren Zeillen finden sich auch überall in dem Zwischenkernraum des Oculomotorius, proximal ziemlich dicht gehäuft.

Es besteht eine Abgrenzung zwischen den Trochleariskernen und den proximal anschliessenden Oculomotoriuskernen, jedoch handelt es sich hierbei (in der Regel wenigstens) nur um eine schmale, zellarme, keine zellfreie Zone. Beim Beginn des Oculomotorius besteht kein grosser Unterschied in der Ausdehnung des Trochlearis- und Oculomotoriuskernes, erst allmählich nimmt letzterer gegen

1) Loc. eit.

9) Loc. cit. 
die Mitte des Kerngebietes hin zu und wird dann proximal wieder ebenso allmählich kleiner.

Sofort kenntlich ist der Beginn des Oculomotorius an dem Auftreten von Fasern im dorsalen Gebiet zwischen den beiden Kernen, welche einen geschlängelten Verlauf nehmen, ja welche förmliche, meist dorsoconcave Schlingen bilden. Siehe Fig. 7 auf Taf. XIV. In dem unteren Bem reich sehen wir, ebenso wie zwischen den Trochleariskernen lediglich Bruchstücke ron Fasern, welche nur ganz kurz in ihrem Verlauf au verfolgen sind.

Ausser diesen von einem Kerngebiet zum anderen ziehenden (sich kreuzenden) Fasern bemerken wir beim Beginn des Oculomotoriuskerngebietes noch eine Anzahl von Zellen im dorsalen Zwischenkernraum, die ganz denen der Hauptkernmasse gleichen und gewissermaassen eine Brücke von einem Kern zum anderen bilden.

Einzelne Ganglienzellen liegen im distalsten Bereich sowohl wie, weiter vorn auch zwischen den Längsbündelquerschnitten, dieselben gehören sicher zum Oculomotoriuskern.

In dem distalsten Bereich verlassen die aus dem Kerngebiet austretenden Faserbündel dasselbe nur zwischen den lateralen, hauptsächlich den dorsalen Längsbündelquerschnitten, nach ca. 20 Schnitten treten auch Fasern in grösserer Zahl in dem Zwischenraum zwischen dem medialen Rande der Längsquerschnitte der rechten und linken Seite aus.

Die sich kreuzenden Oculomotoriusfasern werden nach vorn zu immer spärlicher, ihre Schlingen ragen zum Theil weit ventralwärts in den $Z$ wischenkernraum herein; sie sind jedoch bis in das proximale Drittel des Kernes herein zu verfolgen.

Hingegen werden die ungekrenzten Fasern und deren austretende Faserbündel immer mächtiger, dieselben verlassen mehr und mehr das Kerngebiet durch die medial gelegenen Längsbiundelquerschnitte. Siehe Fig. 8 auf Taf. XIV.

Eine wirkliche Gliederung des Kerngebietes in eine 
grössere Anzahl von Abschnitten existirt nicht, sie wird nur scheinbar durch Fasern hervorgerufen, welche sich schon innerhalb des Kerngebietes zu dickeren Bündeln sammeln.

Im proximalen Kernabschnitt finden wir im dorsomedialen Gebiet des $Z$ wischenkernraumes eine grössere Anzahl dicht gehäufter, kleinerer Ganglienzellen, die die Erinnerung an den sog. kleinzelligen Mediankern Edinger ${ }^{1}$ ) Westphal's ${ }^{2}$ ) (Bernheimer's ${ }^{3}$ ) paarige Mediankerne) wachrufen. Siehe Fig. 1 auf Taf. XV.

Fin unpaarer Mediankern war so gut wie gar nicht gebildet. Grosse Ganglienzellen fanden sich nur beim Beginn des Ocnlomotoriuskernes im dorsalen Bereich des Zwischenkernraumes.

Die aus dem Kerngebiet austretenden Faserbündel nehmen auf ihrem Wege zur Hirnbasis einen S-förmigen Verlauf und vereinigen sich erst vor der Austrittsstelle an der Basis zum Nervenstamm des Oculomotorius.

Die Querschnitte des hinteren Längsbündels nehmen allmählich an Mächtigkeit vom distalen nach dem proximalen Ende zu ab.

Frontalschnittserie durch das Trochlearis- und

Oculomotoriuskerngebiet eines $24 \mathrm{Jahre}$ alten

Jannes nach Weigert. Hierzu Fig. 10-12 auf Taf. XV u. XVI.

Es liegt eine lückenlose Serie von 170 Schnitten vor, welche in der Serie die Nummern 1-170 fübren. Die Schnitte sind durchschnittlich $35-40 \mu$ stark. Es wurde von der distalen nach der proximalen Seite zu geschnitten.

Das Trochleariskerngebiet umfasst 56 Schnitte.

Schnitt 32. Derselbe geht ungefahr durch die Mitte des Trochleariskerngebietes. Der Kerm hat eine nahezu ovale Form und liegt der dorsalen Seite des hinteren Längsbündels an und zwar in einer schwach dorsoconcaven Aushöhlung desselben. - Wir finden hier gleich wie später im Oculomotoriuskern multipolare Zellen von motorischem Typus, die Grösse der Zellen ist in beiden Kerngebieten
1) Loc. cit.
2) Loc. cit.
3) Loc, cit. 
eine etwas wechselnde; im Trochleariskern sind anscheinend die grösseren Zellen etwas mehr vorherrschend. - Die Zellen sind allesammt von einem dichten Netzwerk feinster Fasern umsponnen. Das Kerngebiet wird von einer Anzahl gröberer, kreaz- und quer verlaufendex Fasem durchzogen, welche zum Theil schon innerhalb des Kernes sich zu gröberen Bündeln sammeln. Diese Letzteren nehmen einen gewundenen Verlauf zwischen den Ganglienzellen hindurch, treten zwischen den Quer- und Schrägschnitten des hinteren Längsbündels aus und sind nun nicht weiter zu verfolgen. -Auffallig ist eine Anzahl stark markhaltiger Fasem (gleich denen des hinteren Längsbündels) am dorsalen und medialen Theil des Kernes. - Die Begrenzung des Kernes gegen das hintere Luangsbündel ist keine scharfe, insofern als mehrere Quer- und Schrägschnitte des hinteren Längsbündels stark in die Kernmasse selbst hineinragen Der Kern reicht bis an das mediale Ende der Längsbündelquerschnitte heran. - Der ziemlich grosse Zwischenraum zwischen beiden Kernen wird von einer spärlichen Anzahl schwacher Fasern durchzogen, welche hauptsächlich in dorsoventraler Richtung verlaufen; auch gewahren wir eine Anzahl kleiner Ganglienzellen daselbst.

In den Schnittten aus der Uebergangszone des Trochlearis- in den Oculomotoriuskern finden wir ein dichtes Fasergewirr, in welchem wir nur eine geringe Zahl von Zellen erkennen, es ist somit der Trochleariskern vom Oculomotoriuskern getrennt, aber nicht durch eine zellfreie, sondern nur durch eine schmale zellarme Zone.

Schnitt 59. Mit diesem Schnitte hat sich das Bild wesentlich geändert; wir sind jetzt in distalsten Abschnitt des Oculomotorius. Zwischen beiden Kernen seben wir eine grosse Anzahl von Fasern und Zellen, welche gewissermaassen eine Brücke von einem Kern zum anderen bilden. Die Ausdehnung des Kernes ist noch ziemlich gleich der des Trochleariskernes, die Form ist eine mehr rundliche. Der Kern überragt etwas den medialen Rand der Längsbündelquersehnitte. - Die Abgrenzung des Kernes gegen das hintere Längsbündel ist viel schärfer als heim Trochleariskern.

Die Fasern, welche in dem Zwischenkernraum rorhanden sind, nehmen zum Theil einen gewundenen Vexlauf von einem Kern zum anderen, zum grösseren Theil bilden sie verschieden weit in den Zwischenkernraum hereinragende dorsoconcave Schlingen. Die Fasern mit dem eben beschriebenen Verlauf sind sich kreuzende Oculomom toriusfasern.

Fasern, welche für eine längere Strecke im Zwischenkernraum in dorsoventraler Richtung ziehen und im Winkel der Längsbündelquersehnitte ventral austreten, sind hier nicht vorhanden. Wir sehen in Kerngebiet eine grosse Zahl von Fasern bereits zu Bündeln gesammelt, dieselben treten lateral zwischen den Längsbündelquerschnitten aus, die starkeren Bündel mehr dorsal. Sie strahlen pinselartig aus dem Kerngebiet aus und sind eine Strecke weit nach aussen davon $\mathrm{zu}$ verfolgen.

Zwischen den Querschnitten des hinteren Längsbündels, vereinzelt auch ausserhalb derselben treffen wir eine Anzahl von Ganglienzellen, welche denen des Kernes ganz gleich sind und sicherlich auch zum Oculomotoriuskern gehören, wie man sich leicht bei der 
Durchsicht der Serie und dem Studium des Faserverlaufes überzeugen kann.

In den nächstfolgenden Schnitten nimmt die Ausdehnung des Kerngebietes etwas $\mathrm{zu}$, besonders in dorsolateraler Richtung. Der Zwischenraum zwischen beiden Kemen ist immer noch mit einer Anzahl grösserer Ganglienzellen bevölkert, die jetzt auch etwas weiter ventralwärts zu finden sind.

Neben den grossen Zellen finden sich auch kleinere Zellen; ausschliesslich die letzteren trefien wir in dem Zwischenraum zwischen den medialen Längsbündelquerschnitten ventral vom Kerngebiet.

Die Zahl der zwischen und ausserhalb der Längsbündelquerschnitte gelegenen Ganglienzellen hat erheblich zugenormmen, es kann kein Zweifel bestehen, dass diese Zellen zum Kerngebiet gehören.

Mit der Zunahme des Kerngebietes hat auch die Zahl der gekreuzten Fasern zugenommen und wir sehen jetzt in der Mittellinie eine sehr grosse Zahl von schlingenförmig umbiegenden Fasern in verschiedenen $A b s c h n i t t e n$ des $Z$ wischenkernranmes; dieselben ragen zum Theil schon in den Zwischenraum zwischen den mehr ventral gelegenen Längsbündelquerschnitten herein. Es ist sehr schön zu sehen, dass die einzelnen Fasern sowohl in ab-, als aufsteigender Richtung, sowie im horizontalen Verlauf sich verschieden verhalten; wir sehen verschieden lange und verschieden breite Schlingen.

Neben diesen eben beschriebenen Fasern sehen wir auch solche, die in dorsoventraler Richtung ziemlich weit in die Aushöhlung zwischen den Längsbündelquerschnitten verfolgt werden können (ungekreuzte Fasern?).

Zwischen den seitlichen Lảngsbündelquerschnitten treten Nervenfaserbündel aus und zwar jetzt nicht mehr wie in den distalsten Schnitten die stärkeren Bündel nur zwischen den dorsal gelegenen Querschnitten, sondern auch zwischen den mehr ventral gelegenen. Diese Faserbündel können schon weit in die umgebenden Hirnparthieen hinaus verfolgt werden, sie nehmen einen leicht geschlängelten, lateroventralen Verlauf.

Schnitt 72. Das Kerngebiet hat an Ausdehnung zugenommen und zwar mehr der Breite als der Länge nach. Es bildet ein Oval mit rentral gelegener Spitze; die dorsalen Abschnitte divergiren ziemlich erheblich.

In dem Zwischenkernraum befindet sich auch hier noch eine Anzahl von grossen Ganglienzellen, doch ist die Zahl derselben bereits geringer geworden.

Zwischen den und auch ausserhalb der Längsbündelquerschnitte sehen wir eine grosse Zahl von Zellen des Oculomotoriuskernes, besonders im dorsalen Bereich.

Gekreuzte Fasern sind in grosser $\mathrm{Zahl}$ vorhanden, die Schlingen derselben ragen zum Theil weit in dis Aushöhlung zwischen den ventralen Längsbundelquerschnitten herein.

Die Zahl der dorsorentral (ungekreuzt?) in dem Zwischenkernraum verlaufenden Fasem hat erheblich zugenommen, sie können zum Theil bis an das ventrale Ende der Längsbündelaushöhlung verfolgt werden.

Schnitt 85. Siehe Fig. 10 auf Taf. XV. Die Ausdehnung des Kerngebietes hat wieder etwas zugenommen, es tritt jetzt mebr eine 
Birnform hervor. Die dorsalen Enden divergiren noch stark, die rentralen liegen nahe beisammen. In dem Zwischenraum zwischen beiden Kerngebieten sehen wir nur mehr dorsal eine etwas grössere Zahl von Ganglienzellen, doch kann man über deren Zugehörigkeit zum Kerngebiet etwas im Zweifel sein. Zwischen und ausserhalb der Längsbündelquerschnitte befindet sich eine nicht unerhebliche Anzahl von Ganglienzellen. Es soll betont werden, dass sich diese Zellen auch zwischen den medial gelegenen Längsbündelquerschnitten vereinzelt finden.

Die Zahl der gekreuzten Fasern hat abgenommen, doch sind mit Sicherheit noch welche nachzuweisen und zwar ragen deren sehlingenförmige Umbiegungsstellen zum Theil weit nach abwärts in die Aushöhlung des hinteren Längsbündels herein. Die in $Z$ wischenkernraum dorsoventralwärts - ungekreuzt - verlaufenden Fasern lassen sich jetzt ganz gut bis an das untere Ende der Aushöhlung des hinteren Längsbündels verfolgen.

Die Begrenzungslinie der Längsbündelquerschnitte ist jetzt eine etwas andere als in den distalsten Schnitten. Früher verlief die obere Begrenzungslinie zunächst ganz wenig nach unten abfallend von aussen nach innen und bog dann ziomlich scharf in die directe Richtung nach unten $\mathrm{um}$, jetzt verläuft sie ganz gleichmässig in schräger Richtung von aussen oben nach innen unten.

Schnitt 98. Die Ausdehnung des Kerngebietes in dorsoventraler Richtung hat wieder etwas zugenommen. Breite und Form sind gleich geblieben. Das ventrale Ende ragt fast bis auf den Grund der Ausbuchtung zwischen den Längsbündelquerschnitten herab. In Bezug auf die im Zwischenkernraum zwischen and ausserhalb der Längsbündelquerschnitte gelegenen Zellen hat sich keine irgend wesentliche Aenderung ergeben. - Am dorsalen Ende des Kerngebietes hebt sich eine rundliche Kernmasse etwas von dem übrigen Kerngebiet $a b$, doch handelt es sich hier nicht um eine wirkliche Abtrennung, sondern es bleibt immer die Verbindung durch eine nur etwas spärlicher mit Ganglienzellen bevölkerte Schicht erhalten.

Die Stärke der zwischen den dorsal gelegenen Längsbündelquerschnitten austretenden Faserbündel hat etwas abgenommen, dahingegen hat die Stärke der mehr ventral gelegenen Faserbündel zugenommen. Die austretenden Faserbündel können weit hinaus verfolgt werden, ihre Verlanfsrichtung hat sich nicht geändert. - Die $\mathrm{Zahl}$ der in dorsoventraler Richtung im $Z$ wischenkernraum verlaufenden Fasern hat zugenommen, wir sehen das ventrale Ende der Aushöhlung des hinteren Längsbündels ganz mit markhaltigen Nervenasern ausgefüllt. - Die gekreuzten Fasern haben an Zahl abgenommen.

Schnit 110. An diesem Schnitt und einer grösseren Zahl der Umgebung tritt beiderseits am dorsalen Ende des Kerngebietes ein Haufen von etwas heller aussehenden und kleineren Zellen hervor. Dieser Zellhaufen hat zunächst Mandel- dann Sichelform und zieht schräg von aussen oben nach innen. Zuerst ist er nur am dorsalen Ende des Kerngebietes zu sehen, almählich greift er auch auf den dorsomedialen Theil desselben über. Eine scharfe Abgrenzung gegen das dorsale Ende der Hauptkernmasse ist zwar durch die ver-

v. Graefe's Archiv für Ophthalmologie. XLVII. 2. 
schiedene Färbung, aber nicht in Wirklichkeit gegeben, insofern als stets in dem $Z$ wischenraum zwischen beiden eine Anzahl von Ganglienzellen sichtbar bleibt, welche ganz denen der Hauptkernmasse gleichen. Diese eben beschriebene Zellenanhäufung erinnert sehr an den später zu beschreibenden sogen. Edinger')-Westphal'schen ${ }^{2}$ ) Kern.

Schnitt 122. Siehe Fig. 10 auf Taf. XVI. Die Ausdehnung der Kernmasse ist ziemlich gleich geblieben ebenso die Form. Die Ganglienzellen ragen jetzt bis an das ventrale Ende der Aushöhlung des hinteren Längsbündels heran. - An diesem Schnite und anch schon an einigen rorausgehenden wird num am dorsomedialen Ende der seitlichen Hauptkernmasse eine ovale Anhäufung von heller gefäroten, etwas kleineren Ganglienzellen sichtbar. Zwischen diesen Ganglienzellenhäufchen und der seitlichen Hauptkernmasse zieht ein stärkeres Faserbündel hindurch, wodurch eine Abgrenzung beider Theile bedingt wird. Doch auch hier existirt keine scharfe Abgrenzung, insofern als auch in dem eben genannten Faserbündel eingestreute Ganglienzellen sich finden von dem Typus der Hauptkermmasse. Ventralwärts davon in und zu beiden Seiten der Mittellinie findet sich eine mandelförmige Anhäufung von Ganglienzellen, die ganz den Typus der Zellen der seitlichen Hauptkernmasse tragen. Diese Zellanhäufung ist scheinbar getrennt von den seitlichen Hauptkernen durch zwei starke, dorsoventralwärts ziehende Faserzüge. Man kann jedoch bei starker Vergrösserung ganz gut sehen, dass auch in diesen Faserzügen grosse Ganglienzellen eingestreut sich finden. - In den voraus-

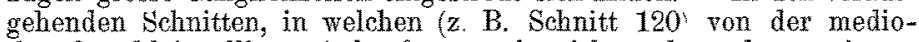
dorsalen kleinzelligen Anhäufung noch nichts oder sehr wenig zu sehen war, fanden wir ebenfalls schon in und neben der Mittellinie die vorhin erwähnte Anhäufung grosser Ganglienzellen; die Abgrenzung gegen die seitlichen Hauptkerne war daselbst noch weniger scharf.

Schnitt 138. Siehe Fig. 11 auf Taf. XVI. Die Form des Kernes ist gleich geblieben, die Ausdehnung desselben hat etwas abgenommen. Die äussere Begrenzungslinie der Hauptkernmasse ist leicht concav, die innere etwas convex. - Die dorsomediale Zellanhäufung ist noch vorhanden, hat aber an Ausdehnung etwas abgenommen In den Schnitten, wo dieselbe die grösste Ausdehnung besass, erreichte sie ungefähr ein Fünftel der Länge der Hauptkernmasse. In den meisten Schnitten ist die kleinzellige Anhäufung auffallend heller gefärbt, doch tritt dieser Unterschied nicht an allen Schnitten in gleichem Grade hervor. An allen Schnitten jedoch fullt auf, dass diese kleinen Zellen viel enger beisammen liegen, viel dichter gehäuft sind, als die Zellen der Hauptkernmasse. In Bezug auf die Grösse finde ich keinen so grossen Untersehied zu den Zellen der Hauptkemmasse, als Bernheimer ${ }^{3}$ ) angiebt. Ich fand beim Menschen die grossen Zellen der Hauptkerne $27,6-4 \dot{b} \mu$, die kleinen Zellen der kleinzelligen Kerne 13,8-20,3 $\mu$.

Der Zwischenraum zwischen diesen kleinzelligen Anhäufungen ist ebenfalls von einer - nicht an allen Schnitten gleich starken Anzahl von Zellen bevölkert.

Etwas nach unten von diesen kleinzelligen Anhäufungen liegt in der Mitte zwischen beiden Hauptkerngebieten der bei der Be-
1) Loc. cit.
2) Loc. cit.
3) Loc. cit. 
schreibung des vorigen Schnittes bereits erwähnte mandelförmige Kern. Derselbe ragt fast bis in den Grund der Aushöhlung des hinteren Längsbündels herein. Zwischen dieser Kernmasse und den seitlichen Hauptkernen sind zwei starke, dorsoventral verlaufende Faserbündel vorhanden. Wir finden an fast allen Schnitten zwischen diesen Faserbündeln vereinzelte Ganglienzellen eingestreut, welche gewissermasssen die Verbindung mit den seitlichen Hauptkernen erhalten und es fraglich erscheinen lassen, ob dieser mandelförmige Kern wirklich einen von der Hauptkernmasse scharf zu trennenden Zellcomplex darstellt oder ob die Abgliederung dieser Kernmasse auch nur wieder lediglicb durch die erwähnten seitlich verlaufenden Faserbundel bedingt ist.

Nahezu in der ganzen Ausdehnung der Längsbündelquer- und Schrägschnitte sehen wir zwischen denselben vereinzelte Ganglienzellen.

Die das Kerngebiet verlassenden stärkeren Faserbündel treten nur noch entsprechend dem ventralen Ende der Aushöhlung des hinteren Längsbündels und den zunächst, gelegenen Querschnitten hindurch. Zwischen den dorsal gelegenen Querschnitten treten nur mehr feine Bündel hindurch. - In dem Zwischenkernraum sind noch ganz vereinzelte Fasern sichtbar, welche einen horizontalen Verlauf nehmen. und leicht dorsoconcare Schlingen bilden.

Schnitt 143. Die Ausdehnung des Kerngebietes hat wieder etwas abgenommen. Die dorsomediale kleinzellige Anhäufung ist fast vollständig verschwunden; von der darunter liegenden mandelförmigen Anhänfung sehen wix nur mehr einige Zellen. - In dem dorsalen Zwischenkerngebiet sehen wir einzelne Zellen von dem Typus der Hamptkernmasse.

Schnitt 156. Siehe Fig. 12 auf Taf. XVI. Die Ausdehnung des Kerngebietes hat wieder abgenommen, die Form ist dieselbe geblieben. An den Längsbündelquerschnitten liegen nur mehr vereinzelte Zellen; die Kernmasse ist gewissermaassen gegen die Mittellinie zusarnmengerückt. Bezüglich der austretenden Faserbündel ist keine Aenderung eingetreten. - Bemerkt sei, dass sowohl an diesem Schnitte als an einer Anzahl der Umgebung die dorsal gelegenen Zellen eine hellere Fürbung darboten.

Yit den nächstfolgenden Schnitten nimmt das Oculomotoriuskerngebiet rasch an Ausdehnung ab. sichtbar.

Dorsolateral wird der sogen. Darkschewitsch'sche ${ }^{1}$ ) Kern

\section{Zusammenfassung und Besprechung der} eigenen anatomischen Untersuchungen über den Trochlearis und Oculomotoriuskern beim Menschen.

Es liegen mir zwei eigene Serien nach Weigert von Erwachsenen, eine lückenlose und eine nicht ganz lückenlose Serie vor, ferner zwei Golgi-Serien menschlicher Embryonen

1) Arch. f. Anatomie u. Phys. Anatom. Abth. 1889. 
(30. und 34. Woche), ausserdem wurden mir zwei Serien von menschlichen Embryonen (8. und 6. Monat) nach Weigert und Pal behandelt von Excellenz v. Koelliker gütigst zur Durchsicht überlassen. Des weiteren wurden mir von Herrn Prof. Rieger drei allerdings ziemlich unvollständige Serien durch das Kerngebiet erwachsener Individuen gütigst zur Verfügung gestellt. Ich spreche Excellenz v. Koelliker und Prof. Rieger hierfür meinen wärmsten Dank aus.

Der Trochleariskern liegt beiderseits in einer dorsoconcaven Aushöhlung des hinteren Längsbündels im Bereiche des vorderen Abschnittes der distalen Vierhügel; er hat eine nahezu ovale Form. Die austretenden Fasern verlaufen nicht direkt zu der Austrittsstelle des N. trochlearis am Velum medullare medium, sondern sie beschreiben während ihres Verlaufes einen doppelten Bogen. - Der Kern besteht aus ziemlich grossen $(40-50 \mu)$ multipolaren Ganglienzellen. Dieselben sind ebenso wie die Zellen des Oculomotoriuskernes von feinen Fasern umsponnen, welche nach v. Koelliker ${ }^{1}$ ) auf Endigungen von Pyramidenfasern, auf sensible zuleitende Elemente zu beziehen sind, deren Herkunft auf den Fasciculus longitudinalis und den Opticus führt.

Proximal schliesst sich direct das Kerngebiet des Oculomotorius an. Zwischen beiden Kernen befindet sich eine mit einer reichlichen Anzahl von Fasern, aber nur wenigen Zellen bevölkerte Zone.

Der Oculomotoriuskern liegt beiderseits im Bereiche des proximalen Vierhügels in einer starken Aushöhlung des hinteren Längsbündels. Ich fand gleich v. Koelliker $\left.{ }^{2}\right)$ eine Länge von etwa $5 \mathrm{~mm}\left(\mathrm{~B} \propto\right.$ rnheimer $\left.^{3}\right)$

1) Handbuch der Gewebelehre des Menschen. 6. Auf. Wilhelm Engelmann. Leipzig 1896.

2) Ibidern.

3) Loc. cit. 
$6 \mathrm{~mm}$ ). Er besteht aus multipolaren mittelgrossen Ganglienzellen.

Im Beginn ist der Oculomotoriuskern kaum grösser als der Trochleariskern, er nimmt aber bis zur Mitte seines sagittalen Durchmessers an Höhe und Breite zu, von da $a b$ wird er dann allmählich wieder kleiner.

Die Hauptkernmasse liegt zu beiden Seiten der Medianlinie. Das dorsale Ende der beiden Kernmassen divergirt, das ventrale Ende convergirt hingegen etwas, so dass der Zwischenkernraum eine dreieckige Gestalt annimmt, mit dorsal gelegener Basis. Ebenso wie ₹. Koelliker') und Bernheimer ${ }^{2}$ ) finde ich, dass an den eigentlichen Oculomotoriuskernen (der seitlichen Hauptkernmasse) Unterabtheilungen nicht existiren.

An einer Auzahl von Schnitten wird durch Faserzüge, welche durch das Kerngebiet ziehen, scheinbar eine Theilung in mehrere Abschnitte hervorgebracht. Diese Trennung besteht jedoch nicht durch eine grössere Anzahl von Schnitten in constanter Weise fort, es handelt sich bestimmt nicht um eine durchgreifende wirkliche Gliederung.

An Golgi-Präparaten, siehe Fig. 13 u. 14 auf Taf. XV, fällt auf, dass die Zellfortsätze sehr lang und weitverzweigt sind, dass sich auch schon im Kerngebiet zarte Stämmchen von Achsencylinderfortsätzen sammeln und durch das Kerngebiet hindurchziehen - alles Momente, welche der Lehre von der scharfen Gliederung und Trennung der einzelnen Ganglienzellenhaufen und der darauf basirenden Lehre von den Kernlähmungen nicht gerade günstig sind.

In dem Zwischenraume zwischen den beiden seitlichen Hauptkernen findet sich ebenfalls besonders im dorsalen Bereich des distalen Kernabschnittes eine nicht unbeträchtliche Zahl von Zellen, die denen der seitlichen Hauptkernmasse gleichen. Siehe Fig. 10 auf Taf. XV. - Ueberall

1) Loc. cit.

2) Loc. cit. 
im Zwischenkernraum des Trochlearis und Oculomotorius finden sich auch kleinere Ganglienzellen, hier und da mehr vereinzelt, hier und da dichter gehäuft.

Zwischen und auch ausserhalb der Längsbündelquerschnitte befindet sich ebenfalls eine oft nicht unbeträchtliche Anzahl von Zellen. Im distalen und dorsalen Kernabschnitt pflegen dieselben etwas zahlreicher zu sein. In Bezug auf die Zahl und Ausdehnung dieser anscheinend versprengten Zellen habe ich individuelle Schwankungen gefunden. Betonen möchte ich, dass ich diese Zellen auch beim Embryo in reichlicher Zahl und ziemlich ausserhalb dex Längsbündelquerschnitte gesehen habe. Sie gehören ganz bestimmt $z \mathrm{u}$ den seitlichen Hauptkernen des Oculomotorius.

Ungefähr mit Beginn der vorderen Hälfte des Kernes fällt im dorsalen Bereich des Zwischenkernraumes beiderseits von der Medianlinie eine hellere ovale Zellanhäufung auf. Siehe Fig. 11 auf Taf. XVI. Thre Zellen sind viel blasser und kleiner (durchschnittlich die Hälfte, nach Bernheimer ${ }^{1}$ ) sind sie vier Mal kleiner als die Zellen der Hauptkernmasse). Diese Zellanhäufung, in welcher wir sofort die Edinger ${ }^{2}$ - West $p$ hal'schen ${ }^{3}$ ) kleinzelligen Mediankerne erkennen, sind weder in der Medianlinie scharf voneinander getrennt, noch auch von der Hauptkernmasse. $Z$ wischen diesen beiden Kermen treffen wir eine verschieden grosse Zahl von kleineren Zellen, ebenso zwischen diesen und den Hauptkernen einzelne grössere und kleinere Zellen.

Dieser sogen. kleinzellige Mediankern steht in manchen Schnitten in Verbindung mit dem sogen. kleinzelligen Lateralkern von Westphal ${ }^{4}$ ). Betreffs der genaueren Schilderung verweise ich auf die erschöpfende Darstellung dieses Autors.
1) Loc. cit.
9) Loc. cit.
8) Loe. cit.
4) Loe, eit. 
Die Verschiedenheit der Zellen dieser Kerne im Vergleich zum Oculomotoriushauptkern, der Umstand, dass weit im Umkreis dieselben Zellen -- nur etwas weniger dicht gehäuft - sich finden, der Umstand, dass ich bei meinen experimentellen Untersuchungen nie Veränderungen in diesen Zellen fand, lässt mir die Berechtigung, fragliche Kerne dem Oculomotoriuskern zuzuzählen, etwas zweifelhaft erscheinen. Bei den von mir vergleichshalber untersuchten Thieren finde ich an den betreffenden Stellen die gleichen Zellen; nur sind sie zum Theil nicht so dicht gehäuft, und sehen deshalb (?) auch etwas dunkler aus und heben sich nicht so scharf von den umgebenden Parthieen ab, wo ganz die gleichen Zellen auf weite Strecken sich finden.

Der Centralkern Perlia's ') (Bernheimer's') unpaarer Mediankern) tritt in meiner Serie sehr schön hervor. Siehe Fig. 11 auf Taf. XVI. Er tritt ungefähr gleichzeitig mit den kleinzelligen Mediankernen in meiner Serie auf.

Den Faserverlauf ${ }^{3}$ ) betreffend konnte ich Folgendes feststellen: In dem distalsten Bereich des Kerngebietes sind fast nur gekreuzte Fasern vorhanden, dann kommen gekreuzte und ungekreuzte Fasern untermischt vor, je mehr proximal der Schnitt liegt, umso mehr prävaliren die ungekreuzten über die gekreuzten Fasern, in der proximalen Hälfte verlaufen fast nur ungekreuzte Fasern.

Die Kreuzung der Fasern geht folgendermaassen vor sich: Aus den verschiedenen Abschnitten des Kernes der einen Seite kommen Fasern und laufen an der medialen Seite des Kernes zusammen. Nun ziehen sie entweder in

1) Loc. cit

2) Loc. cit.

3) Ausserordentlich schöne und klare Bilder sah ich bezüglich des Faserverlaufes an den mir von Excellenz v. Kaelliker gütigst zur Verfügung gestellten zwei Serien menschlicher Embryonen. 
geschlängeltem Verlauf von einem Kern zum anderen oder -w. und so ist es meistens der Fall - sie bilden verschieden lange und breite dorsoconcave Schlingen nahezu im ganzen Bereich des Zwischenkernraumes. Siehe Fig. 10 auf Taf. XV. Nach dieser Schlingenbildung gelangen sie in den Kern der anderen Seite, strahlen wieder etwas auseinander, winden sich zwischen den Ganglienzellen hindurch und treten zu Bündeln vereinigt $z$ wischen den mehr dorsal gelegenen Längsbïndelquerschnitten hindurch.

Die ungekreuzten Wurzelfasern, siehe Fig. 11 u. 12 auf Taf. XVI, treffen wir unvermischt nur im proximalen Theil des Kernes und zwar vornehmlich im medialen Kernabschnitt. Die Hauptmasse dieser Fasern verläuft in der ganzen Breite des Zwischenkernraumes in ziemlich gerader dorsoventraler Richtung. Somit tritt die grösste Anzahl der ungekreuzten Fasern ventral im Winkel des Längsbündelquerschnittes aus, ein kleinerer Theil der Fasern verlässt das Kerngebiet auch zwischen den nächstgelegenen Längsbündelquerschnitten.

Ausserhalb des Kerns verlaufen die gekreuzten Fasern zuerst nach aussen und etwas nach unten, hierauf in leicht convexem Bogen etwas nach hinten und abwärts, dann wieder nach vorne, um schliesslich in einem gegen die Mittellinie zu leicht convexen Bogen zur Austrittsstelle des gemeinschaftlichen Nervenstammes an der Hirnbasis hinzuziehen. An dem rothen Kerne ziehen sie aussen und unten rorbei.

Die ungekreuzten Fasern nehmen einen bald mehr, bald weniger stark geschlängelten, hier und da S-förmigen Verlauf durch den rothen Kern und an dessen Seiten vorbei. Sie verlaufen ebenso wie die gekreuzten Fasern bis fast zur Hirnbasis getrennt in einzelne Bündel.

Die Querschnitte des hinteren Längsbündels nehmen von hinten (distal) nach rorn (proximal) an Mächtigkeit allmählich ab. Es endet sicher eine Anzahl der Fasern desselben in den Augenmuskelkernen. 
Zusammenfassung der eigenen anatomischen Befunde beim Menschen, Affen, bei der Katze und beim Kaninchen, sowie vergleichende Betrachtung mit den Befunden anderer Forscher.

Es ist mir im Interesse der Kürze meiner Abhandlung erlassen, in diesem Abschnitt eine zusammenfassende Literaturübersicht zu geben, da die vorliegende Literatur erst vor kurzer Zeit in v. Koelliker's Handbuch der Gewebelehre und in Bernheimer's Monographie: Das Wurzelgebiet des Oculomotorius beim Menschen, gebührend berücksichtigt wurde.

In der allerletzten Zeit ist eine Abhandlung von W. v. Bechterew ${ }^{1}$ ) über unser Thema erscheinen. Seine Ergebnisse sind folgende: Auf Grund der Untersuchung einer Anzahl fötaler menschlicher Gehirne und solcher von Neugeborenen gelangte er zur Ueberzeugung, dass der N. oculomotorius eigentlich vier Kerne besitzt: zwei grössere, von welchen der eine paarig, der andere unpaar ist, und zwei kleinere oder accessorische, beide paarig. - Zu den ersteren gehört vor Allem der unter dem vorderen $Z$ weihügel zu beiden Seiten der Mittellinie befindliche Hauptkern.

Im Niveau des mittleren und vorderen Drittels der Hauptkerne wird man noch eines besonderen, grosse Nervenzellen beherbergenden, unpaaren, medialen Kernes gewahr. Derselbe liegt in der Mittellinie zwischen den vorhergehenden Kernen und ist im Vergleich zu den letzteren viel kleiner.

Ferner findet sich dorsal, theils aber auch dorsal und nach aussen von den Hauptkernen ein kleines paariges, wie es scheint, nicht ganz von den letzteren abgesondertes Gebilde.

1) Archiv f. Anatomie u. Entwicklungsgesehichte. 5. a. 6. Heft. Jahrgang 1897. S. 307. 
Nach vorn von den Hauptkernen liegt endlich ein kleiner paariger Kern im Querschnitt von abgerundeter Form zu beiden Seiten der Mittellinie. Letztere bezeichnet er als accessorische Kerne.

Den oberen Oculomotoriuskern (Darkschewitsch 1)scher Kern) rechnet v. Bechterew nicht zum Oculomotorius. - v. Bechterew unterscheidet gekreuzte und ungekreuzte Fasern.

Die beigegebenen Abbildungen sind leider grossentheils bedauerlich schlecht und tragen wenig zur Orientirung bei.

Von den früheren Autoren muss ich wohl ganz kurz Gudden's ${ }^{2}$ ) und Perlia's ${ }^{3}$ ) Erwähnung thun; ersterer hat zuerst die partielle Kreuzung beim Kaninchen, letzterer beim Menschen nachgewiesen.

Als wichtigstes Ergebniss meiner eigenen Untersuchungen erscheint mir die Feststellung, dass eine so scharfe und weitgehende Gliederung im Oculomotoriuskern des Menschen, wie sie von manchen Autoren, z. B. Perlia ${ }^{4}$, angegeben wird und wie sie die Lehre von den Kernlähmungen voraussetzte und verlangte, entschieden nicht existirt. $Z u$ beiden Seiten der Mittellinie liegt ein grosser ungegliederter Hauptkern. In Bezug hierauf finde ich mich in voller Uebereinstimmung mit v. Koelliker ${ }^{5}$ ), Bernheimer ${ }^{6}$ ) und v. Bechterew ${ }^{7}$ ).

Ebensowenig wie beim Menschen existirt beim Kaninchen, der Katze und dem Affen eine weitgehende, wirkliche Gliederung des seitlichen Hauptkernes in eine Anzahl Unterabtheilungen.

Ich habe bei der Beschreibung meiner Befunde aus-

1) Loc. cit.

7) Gesammelte und hinterlassene Abhandlungen, herausgegeben von Grashey. XXXI., Angenbewegungsnerven.

3) Loc. cit. 4) Loc. cit. 5) Loc. cit. 5) Loc. cit. 7) Loc. eit. 
führlich auseinandergesetzt, auf welche Weise eine scheinbare Gliederung hervorgerufen wird. Es sei hier einfach darauf verwiesen.

Den Zwischenraum zwischen dem rechten und linken Kern fand ich in den mir vorliegenden Serien verschieden breit, am breitesten beim Affen, weniger breit beim Menschen und Kaninchen, eigentlich gar kein Zwischenraum fand sich in den mir vorliegenden zwei Weigertserien der Katze. - Man vergleiche in dieser Beziehung die beigegebenen Abbildungen auf Taf. XIII-XVI.

Auch an den Thioninserien fand ich in den distalen und mittleren Parthieen des Kernes fast gar keinen Zwischenkernraum. Im proximalen Drittel besteht dorsal und in der Mitte ein $Z$ wischenraum, ventral auch hier so gut wie keiner.

Zu Beginn des Oculomotorius (distal) fand ich sowohl beim Menschen, als beim Affen und der Katze eine etwas reichlichere Anhäufung von grossen Ganglienzellon im dorsalen Bereich des Zwischenkernraumes. Beim Kaninchen waren nur wenige solcher Zellen vorhanden. Diese Zellen gleichen vollständig denen der Hauptkernmasse, wovon man sich an Thionin- oder Toluidinblaupräparaten überzeugen kann (vgl. die Abbild. 1, 4, 7 u. 10 auf Taf. XIII-XV).

Abgesehen von diesen grossen Ganglienzellen sind noch kleinere vorhanden und zwar finden sich diese kleinen Zellen im ganzen Bereich des $Z$ wischenkernraumes und zwar nicht bloss des Oculomotorius sondern auch des Trochlearis, sie finden sich auch dorsal, distal und proximal von den Kerngebieten in wechselnder Zahl und verschieden dicht gehäuft. Es sind dies dem Aussehen nach ganz die gleichen Zellen, die wir später bei der Besprechung der kleinzelligen Edinger ${ }^{1}$-Westphal'schen ${ }^{2}$ ) Kerne begegnen. Gegen

1) Loc. cit.

?) Loe. cit. 
die Mitte des Kerngebietes zu werden die grossen Ganglienzellen im Zwischenkernraum beim Menschen selten, beim Affen und Kaninchen sind fast gar keine mehr zu. sehen, dahingegen nehmen sie bei der Katze an Zahl zu. und sind nahezu in der ganzen Ausdehnung des $Z$ wischenkernraumes vorhanden.

Eine bestimmte Function scheint diesen Zellen nicht zuzukommen, ihr Vorkommen ist, wie gesagt, ein wechselndes. Es scheint kein Grund vorhanden zu sein, ihnen einen besonderen Namen zu geben.

Im vorderen Theil des Kerngebietes begegnen wir im sogenannten Zwischenkernraum in und zu beiden Seiten der Medianlinie diesen grossen Ganglienzellen noch einmal und zwar in etwas auffallender Gruppirung. Wie aus der Abbild. 11 auf Taf. XVI des Kerngebietes beim Menschen hervorgeht, kommt es zu einer mandelförmigen Zellanhäufung (Perlia's ') Centralkern, Bernheimer's ${ }^{2}$ ) unpaarer Mediankern). Es ist dies ein beim Menschen anscheinend constant vorkommender Befund. - Die Abgrenzung gegen die seitlichen Hauptkerne wird verschieden angegeben und abgebildet. Ich fand keine scharfe Abgrenzung gegen die seitlichen Hauptkerne. $\mathrm{Zu}$ beiden Seiten verlaufen zwei starke Faserzüge in dorsoventraler Richtung, und durch diese wird die Abgliederung scheinbar hervorgebracht. Ich sah zwischen dieser mandelförmigen Kernanhäufung und den seitlichen Hauptkernen keine zellfreie, sondern nur eine zellarme Zone. Ebenso wie in den Seitenhauptkernen wird auch hier durch die Faserzüge keine wirkliche, sondern eine scheinbare Abgliederung hervorgebracht. In dieser Auffassung werde ich bestärkt durch den Umstand, dass etwas distal von dem sogenannten Centralkern Perlia's auch die Mittellinie fast vollständig von grossen Ganglien-

\footnotetext{
1) Loc, cit.

2) Loo. cit.
} 
zellen bevölkert ist, hier sind aber nicht (in meiner Serie des Erwachsenen) die starken dorsoventralziehenden Faserbündel vorhanden, hier kommt man daher nicht zu der Annahme eines abgegliederten Kernes. In den zwei mir vorliegenden Weigertserien der Katze ist einmal durch zwei starke Faserbündel ein sogen. Centralkern schön ausgebildet, in der anderen Serie treten die starken Faserzüge nicht hervor und hier hat dann auch keine Abgliederung der im $Z$ wischenkernraum vorhandenen grossen Ganglienzellen statt (vgl. die Abbild. 5 u. 6 auf Taf. XIV). Beim Kaninchen und Affen finde ich diesen sogen. Centralkern fast gar nicht ausgebildet. In Bezug auf den Affen steht mein Befund im Gegensatz zu Bernheimer's Angabe. Hat dieser Kern wirklich die bestimmte Function, die Bernheimer annimmt, dann müsste er doch bei all diesen Thieren, besonders beim Affen, wenigstens deutlich vorhanden sein. Sehwankungen in seiner Grösse dürften selbstredend vorkommen.

Ausser diesen im $Z$ wischenkernraum vorhandenen grossen Ganglienzellen fand ich auch grosse Ganglienzellen ausserhalb der Hauptkernmasse zwischen und ausserhalb der Längsbündelquerschnitte, besonders im distalen und dorsalen Bereich. (Bernheimer nennt diese Zellen "Lateralzellen"). Beim Menschen fand ich diese Zellen in grosser Zahl und zum Teil in weiter Ausdehnung, ziemlich zahlreich fand ich diese Zellen auch beim Kaninchen, spärlicher beim Affen und nur vereinzelt bei der Katze. Tch habe mich übrigens selbst überzeugen können - sowohl beim Menschen als bei den von mir untersuchten Thieren, dass in dieser Beziehung Schwankungen vorkommen. - Gleich v. Koelliker ${ }^{1}$ ) und Bernheimer ${ }^{2}$ ) bin auch ich schon auf Grund der anatomischen Untersuchung der bestimmten Ueberzeugung,

5) Los. cit.

2) Loc. cit. 
dass diese Zellen zu den Oculomotoriuskernen gehören und gewissermaassen Ausläufer derselben darstellen. - Bernheimer's ${ }^{1}$ ), Schwabe's ${ }^{2}$ ) und meine experimentellen Ergebnisse haben diese Annahme vollständig bestätigt und absolut sicher gestellt.

Auf die sogen. kleinzelligen Edinger $\left.{ }^{3}\right)$-Westphalschen $\left.^{4}\right)$ Mediankerne (Bernheimer's') paarige Mediankerne) ist bei meinen Abbildungen besonders Ruicksicht genommen, da in dieser Beziehung die Meinungen noch ziemlich auseinandergehen. Meine Befunde stimmen im Grossen und Ganzen mit denen v. Koelliker's ${ }^{5}$ ) und Anderer überein, stehen aber im Widerspruch zu den Resultaten Bernheimer's ').

Ich habe in Bezug auf diese Kerne folgende Beobachtungen gemacht: Beim Menschen finde ich ungefähr entsprechend dem Beginn der proximalen Kernhälfte nicht ganz bis an das proximale Ende heranreichend, in dem Raum zwischen den beiden seitlichen Hauptkernen, dorsal zu beiden Seiten der Medianlinie eine bei Weigertpräparaten sofort in die Augen springende dichte Anhäufung kleiner Zellen. Seitlich stehen dieselben durch eine zellärmere Zone mit den Hauptkernen in Verbindung, die Trennung wird nur deutlicher durch ein stärkeres Faserbündel markirt. In der Mittellinie hängen sie durch eine spärlichere Zellansammlung zusammen; auch hier wird ihre scheinbare Trennung durch einen starken Faserzug deutlich gemacht.

Diese Zellanhäufung fand ich, etwas mehr dorsal, auch

a) Loc. cit.

2) Ueber die Gliederung des Ocnlomotoriushauptkernes und die Lage der den einzelnen Muskeln entsprechenden Gebiete in demselben. Neurol. Centralbl. 1897. Nr. 17. S. 792.

3) Loc. cit.

4) Loc. cit.

5) Loe, cit. 
beim Affen, bei der Katze und bei dem Kaninchen. Sie tritt bei diesen Thieren nicht so deutlich hervor, wie bei den Serien der erwachsenen Menschen. Details übergehe ich hier und verweise auf meine durchaus naturgetreuen Abbildungen und auf die Beschreibung S. 342, 347, 355 u. 362. Bernheimer irrt bei seiner Angabe, dass diese sogen. kleinzelligen Mediankerne beim Kaninchen nicht vorhanden seien.

Beim Menschen geht dieser dorso mediale kleinzellige Kern (Edinger ${ }^{1}$ )-Westphal ${ }^{2}$ )) an manchen Schnitten direct über in den kleinzelligen dorsolateralen Kern (Westphal ${ }^{2}$ ). Letzterer tritt ungefähr gleichzeitig mit dem dorsomedialen Kern in die Erscheinung und bietet dasselbe Aussehen. Zuerst repräsentirt er sich als ein schmales helleres Band, das sich horizontal am dorsalen Ende des seitlichen Hauptkernes hinzieht. In proximaler Richtung nimmt diese kleinzellige helle Anhäufung an Ausdehnung $\mathrm{zu}$, steigt etwas dorsolateral an und nimmt allmählich eine mehr länglich ovoide Form an. Ich finde diese Anhäufung kleiner Zellen über das proximale Ende des Oculomotoriushauptkernes binaus.

Beim Affen, dem Kaninchen und bei der Katze tritt dieser dorsolaterale Kern kaum hervor. Wir sehen zwar auch bei diesen Thieren kleinere Ganglienzellen direct anschliessend an das dorsale Ende der Oeulomotoriushauptkerne, sie sind nur etwas weniger dicht gehäuft und zeigt dieser Complex keine so helle Farbe, weshalb er weniger hervortritt.

Nach den Ergebnissen der vergleichend anatomischen Untersuchung habe ich nicht den Eindruck gewinnen können, dass der sogen. kleinzellige Lateralkern $($ Westphal')) ein Bestandtheil des Oculomotoriuskernes
1) Loc. cit.
2) Loc. cit. 
ist. Die experimentelle Forschung hat diese Anschauung bestätigt.

Bei den von mir untersuchten Thieren verlieren sich die sogen. kleinzelligen Mediankerne ganz allmählich in die etwas zellärmere Umgebung.

An Thionin- und Toluidinblaupräparaten kann man sich einmal davon überzeugen, dass die Zellen der sogenannten kleinzelligen Kerne ein ganz anderes A ussehen darbieten, wie die Zellen der Oculomotorius-, Trochlearis- und Abducenskerne (welche alle gleich aussehen), weiterhin auch davon, dass die Zellen der sogenannten kleinzelligen Median- und Lateralkerne, sowie die dorsal anschliessenden Zellen ein gleiches Aussehen darbieten, somit doch auch wahrscheinlich eine gleiche Function haben. Ausserdem kann man sich an Thionin- und Toluidinblaupräparaten leicht davon überzeugen, dass diese kleineren Zellen in dem ganzen Bereich des $Z$ wischenkernraumes des Oculomotorius und Trochlearis, ferner, dass sie auch distal vom Trochlearisende und proximal vom Oculomotoriusende vorhanden sind.

Auf Grund der im Vorstehenden angeführten Thatsachen bin ich gleich v. Koelliker zur Zeit der Ueberzeugung, dass wenig Berechtigung besteht, diese kleinzelligen Kerne (Lateral- und Mediankerne) als Oculomotoriuskerne anzusehen. Es sind hier noch weitere Erfahrungen zu sammeln.

Von vornherein unwahrscheinlich muss ich die Behauptung Bernheimer's ${ }^{1}$ ) bezeichnen, dass die kleinzelligen Mediankerne das Centrum für den Musculus sphincter pupillae, der grosszellige Mediankern (Perlia's²) Centralkern) das Centrum für den Musculus ciliaris bilden. Was für Gründe sollte die Verschiedenheit der Zellen,

1) Loc. cit. u. Zur Kenntniss der Localisation. im Kerngebiet des Oculomotorius. Vorl. Mitth. Wiener klin. Wochenschr. Nr. 5. 1896 u. v. Graefe's Arch. f. Ophthalm. XLIV. 3. Abth. S. 481.

2) Loc. cit. 
wie sie zwischen diesen Kernen besteht, haben, wenn ihnen wirklich die genannte Function zukäme?

Zur weiteren Klärung der eben behandelter Frage muss ich hier kurz das Ergebnis meiner experimentellen Untersuchungen vorausnehmen.

Bei Entfernung des Musculus sphincter pupillae und des Musculus ciliaris konnte ich bei dem Kaninchen und bei der Katze überhaupt keine Veränderungen im Oculomotoriuskern - weder an den grossen, noch an den kleinen Ganglienzellen - nachweisen. Beim Affen ergab eine Exenteratio orbitae keine Veränderung in den kleinzelligen Mediankemen. - Beim Kaninchen konnte auch Bernheime ${ }^{1}$ ) keine Veränderungen im Oculomotoriuskern nachweisen, bestätigt somit meine Resultate. Allerdings nimmt er irrthümlicher Weise an, dass beim Kaninchen diese kleinzelligen Kerne nicht vorhanden seien. - Gleich mix ${ }^{2}$ ) und Bernheimer ${ }^{1}$ konnten anch $\mathrm{Massaut}^{3}$ ) und Schwabe ${ }^{4}$ ) beim Kaninchen nach Entfernung des Musc. sphincter pupillae und des Musculus ciliaris keine Veränderungen im Oculomotoriuskern sehen. Massaut ${ }^{3}$ ) hätte allerdings höchstwahrscheinlich auch keine Veränderungen gesehen, selbst wenn Schwabe's ${ }^{4}$ ) und meine bezüglichen Experimente positiv ausgefallen wären, da seine Versuchsdauer zu lang war.

Bernheimer ${ }^{1}$ ) hat an Affen (Rhesusaffen) zweimal die Exenteratio bulbi ausgeführt and darnach beide Male Veränderungen in dem kleinzelligen Mediankern derselben Seite nachweisen können, sowie in der entsprechenden Hälfte des grosszelligen Mediankerns.

Ich wage nicht, auf Grund meines einen Versuches beim Affen, Bernheimer's Befunde für unrichtig zu be-

1) Loc. cit. a. Zur Kenntniss der Localisation in Kerngebiet des Oculomotorius. Vorl. Mitth. Wiener klin. Wochenschr. Nr. 5. 1896 u. v. Graefe's Arch. f. Ophthalm. XLIV. Bd. 3. Abth. S. 481.

a) Loc. cit.

s) Arch. f. Psychiatrie. XXVIII. Bd: Heft II. S. 432. 1896.

4) Loc. cit.

จ. Graefe's Arehiv für Ophthalmologie. XLVII. 2. 
zeichnen, im gewissen Sinne jedoch widerspricht sich nach meinen Befunden Bernheimer selbst, da er beim Kaninchen nach der Exenteratio bulbi ein negatives Resultat bekam und der gewiss irrthümlichen Annahme ist, dass die Kleinzelligen Mediankerne beim Kaninchen ganz fehlen.

Schwabe's und meine Resultate nach der Exenteratio bulbi dürften beweisen, dass die Zellen des Oculomotoriuskernes nicht direct auf die interioren Muskeln einwirken. Könnten nun die genannten Zellgruppen nicht indirect auf die genannten Muskeln einwirken? Nach den obigen Auseinandersetzungen muss ich diese Annahme zur Zeit für unbewiesen halten, womit ich natïrlich nicht leugnen will, dass überhaupt ein indirecter Einfluss des Oculomotoriuskernes auf die interioren Augenmuskeln statt hat. Sind die kleinzelligen Kerne wirklich Bestandtheile des Oculomotoriuskernes, so erscheint es mir wahrscheinlicher, dass sie sow ohl den Sphinkter pupillae als den M. ciliaris innerviren. Ich hoffe, bald auf diese Frage zurückkommen zu können.

Weitere Untersuchungen hätten über die Zugehörigkeit der kleinzelligen Mediankerne zum Oculomotoriuskern und ihre Beziehungen zum Sphinkter pupillae definitiv zu entscheiden.

Bezüglich des sogenannten oberen Oculomotoriuskernes (Darkschewitsch) bin ich gleich v. Koelliker, Bernheimer u. A. der Ansicht, dass dieser Kern nicht zum Oculomotorius gehört. Die Zellen dieses Kernes sind etwas kleiner als die des Oculomotoriuskernes, sehen aber auch bei Weigertscher Behandlung dunkel aus. - Die Lage dieser Zellgruppe beim Menschen ist bekannt und habe ich den Befunden anderer Autoren in dieser Beziehung nichts hinzuzufügen.

Beim Affen fiel mir auf, dass von der Gegend des Darkschewitsch'schen Kernes bis zum Oculomotoriuskern hin zwischen den Längsbündelquerschnitten sich eine bald grössere, bald geringere Anzahl von Zellen findet, die denen des Darkschewitsch'schen Kernes gleichen und gewisser- 
maassen eine Verbindung beider Kerne darstellen (siehe Abb. 9 auf Taf. XV. - Aehnlich liegen die Verhältnisse auch beim Kaninchen und bei der Katze, etwas anders beim Menschen (siehe Abb. 12 auf. Taf. XVI).

Beim Affen und bei der Katze fällt im proximalen Kernabschnitt eine kleine Anhäufung von Garrglienzellen in und zu beiden Seiten der Mittellinie in geringer Entfernung vom dorsalen Ende des Kerngebietes auf. Die Zellen sind etwas kleiner als die der Oculomotoriushauptkerne, jedoch grösser als die der sogenannten kleinzelligen Oculomotoriuskerne. Sie haben bei der Weigertbehandlung dieselbe braune Farbe angenommen wie die grossen Zellen des Oculomotoriushauptkernes. Beziehungen zum. Oculomotorius konnte ich nicht feststellen.

In dem einen der eingangs erwähnten paarigen accessorischen Kerne v. Bechterew's ${ }^{1}$ ) ist wohl nach der Abbildung der sogen. kleinzellige Lateralkern Westphal's ${ }^{2}$ ) zu erblicken; bezüglich des anderen paarigen accessorischen. Kernes habe ich aus der beigegebenen Abbildung kein rechtes Urtheil mir bilden können. Beide Kerne haben wohl sicher nichts mit dem Oculomotorius zu thun.

Hinsichtlich des Faserverlaufes hat sich kein principieller Unterschied bei der vergleichend anatomischen Untersuchung ergeben.

Sowohl beim Menschen als beim Affen, dem Kaninchen und der Katze giebt es gekreuzte und ungekreuzte Oculomotoriusfasern ${ }^{3}$ ).

Die gekreuzten Fasern sind distal weitaus zahlreicher, ja beim Menschen, beim Affen und dem Kaninchen kamen in den Schnitten aus dem distalsten Kernabschnitt nur gekreuzte Fasern zur Beobachtung. Je mehr wir in dem

1) Loc, cit.

2) Loc. cit.

3) Bemerkt sei hier, dass auch für Vögel die theilweise Kreuzung der Oculomotoriusfasern nachgewiesen ist - van Gehuchten. 
Kerngebiet nach vorn vorrïcken, umso geringer wird die Zahl der gekreuzten Fasern und im proximalen Drittel sind gar keine oder fast gar keine gekreuzten Fasern mehr vorhanden. Die Zahl der gekreuzten Fasern war in meinen Serien am zahlreichsten bei der Kazte, etwas weniger waren beim Merschen und Affen vorhanden, am wenigsten zahlreich waren sie beim Kaninchen.

Der Verlauf der gekreuzten wie auch der ungekreuzten Fasern ist bei der Serienbeschreibung genau angegeben und verweise ich darauf.

Ueber geringe Variationen in der Art des Verlaufes geben die beigegebenen Abbild. 1-12 auf Taf. XIII-XVI am besten Aufschluss.

Die das Kerngebiet verlassenden Bündel der gekreuzten und ungekreuzten Fasern verlaufen bis zur Hirnbasis getrennt. Dieser Umstand ist von Bedeutung und wäre darauf sowohl bei der experimentellen Forschung als bei pathologischanatomischen Untersuchungen, als auch bei der klinischen Betrachtung von Augenmuskellähmungen in Zukunft zu achten.

Die Längsbündelquerschnitte nehmen vom distalen nach dem proximalen Ende zu allmählich an Stärke ab. Verfasser ist gleich v. Koelliker, Bernheimer, Perlia, v. Bechterew u. A. der Ansicht, dass eine grosse Zahl von Fasern des hinteren Längsbündels in dem Trochlearis und Oculomotoriuskem endet. Es sei hier auf die Thatsache aufmerksam gemacht, dass das hintere Längsbündel die Fortsetzung des Vorderstranggrundbündels des Rückenmarkes darstellt. Es scheint mir nicht unwahrscheinlich, dass diese Bahn auch für die reflectorische Pupillenbewegung in Frage kommt.

Bezüglich des Trochleariskernes haben ebenfalls bis zu den letzten Jahren beträchtliche Widersprüche bestanden. Westphal und Siemerling haben einen grosse Zellelemente enthaltenden, im Niveau des hinteren Zweihügels, am cèntralen grauen Canal dorsalwärts und dorsolateralwärts vom hinteren Längsbündel befindlichen Kern, als den Hauptkern 
des Trochlearis angesehen. Andererseits ist von Westphal. die rundliche Gruppe kleiner Zellelemente, welche dem caudalen Theil des oben beschriebenen Kernes anliegt, als hinterer kleinzelliger Trochleariskern beschrieben worden. Gegen diese Annahme hat sich in letzterer Zeit $\mathrm{K}$ ausch ausgesprochen; er hält die distal an den Oculomotoriuskern anschliessende Zellgruppe (den Nucleus centralis posterior n. oculomotorii einiger Autoren) für den Trochleariskern. Dieser Annahme ist neben einigen anderen auch v. Koelliker und v. Bechterew.

Verfasser ist auf Grund seiner vergleichend-anatomischen Untersuchungen ebenfalls vollständig der gleichen Ansicht. Hätte überhaupt noch ein Zweifel bestanden, so wäre dieser durch das Ergebniss der experimentellen Untersuchung zerstreut worden.

Nach des Verfassers Untersuchungen liegt zwischen dem Oculomotoriuskern und dem distal anschliessenden Trochleariskern ein geringer, nicht immer ganz gleich grosser, in der Regel nicht zellfreier, sondern nur zellarmer Zwischenraum. Sowohl meine vergleichend-anatomischen Befunde als. die Ergebnisșe meiner Experimente führen mich zu der Annahme, dass keine so scharfe Trennung zwischen Oculomotorius- und Trochleariskern besteht, wie von anderer Seite angenommen wird. - Der Trochleariskern liegt in einer ziemlich starken dorso concaven Aushöhlung des hinteren Längsbündels. Die Quer- und Schrägschnitte desselben springen theilweise etwas in das Kerngebiet herein und bilden zum Theil nicht bloss die ventrale, sondern auch die laterale und dorsale Begrenzung. Er besteht gleich dem Oculomotoriuskern aus mittelgrossen multipolaren Zellen, die in einem dichteren Fasernetzwerk liegen. Die Zahl der grösseren Zellen wiegt vielleicht gegenüber dem Oculomotoriuskern etwas vor.

Die Form war beim Menschen und bei der Katze mehr ovoid, beim Affen und beim Kaninchen eine ziemlich runde.

Die Länge des Trochleariskern betrug stets ungefähr $1 / 3$ der Ausdehnung des Oculomotoriuskernes. 
Ziemlich allgemein wird angenommen, dass die Trochlearisfasern eine totale Kreuzung innerhalb des vorderen Marksegels erfahren. - Für das Kaninchen kann ich mit aller Bestimmtheit angeben, dass der weitaus grösste Theil der Fasern sich kreuzt, dass ein kleiner Theil jedoch ungekreuzt verläuft. Bei der Katze fand ich ebenfalls die gekreuzten Fasern in der Ueberzahl, die ungekreuzten waren etwas zahlreicher als beim Kaninchen. Auch beim Affen fand ich gekreuzte und ungekreuzte Fasern, erstere in der Ueberzahl. Da ich jedoch von dem Affen nur eine Serie besitze, will ich diese Angabe nur mit aller Reserve ausgesprochen haben.

Wie die Verhältnisse beim Menschen liegen, wage ich auf Grund der mir vorliegenden Weigert- und GolgiSerien nicht zu entscheiden, doch sollte man wohl füglich annehmen dürfen, dass, nachdem die partielle Kreuzung für Sängethiere erwiesen ist, eine solche wohl auch für den Menschen anzunehmen ist. Weitere Untersuchungen hätten jedoch darüber erst definitiv zu entscheiden.

Auch auf den Abducenskern erstreckten sich meine Untersuchungen. Derselbe liegt im dorsalen Brückentheil etwas lateroventral vom Facialisknie. Er besteht aus genan denselben Zellen wie der Trochlearis- und Oculomotoriuskern. Seine Länge betrug beim Kaninchen und bei dem Affen ca. $1 \mathrm{~mm}$, bei der Katze etwas über $1 \mathrm{~mm}$, beim Menschen ca. 1,5 mm.

\section{Pathologisch-anatomischer Abschnitt.}

Die Ergebnisse vorliegender pathologisch-anatomischer Untersuchungen für die Localisationsfrage im Oculomotoriuskern.

Kahler ${ }^{1}$ ) und Pick ${ }^{1}$ ) haben die erste mikroskopische Untersuchung des Oculomotoriuskernes in zwei Fällen von Kernlähmung ausgeführt. Auf Grund der Befunde waren sie der Meinung,

1) Arch. f. Psychiatrie. Bd. X. 
dass von den hinteren lateralen Bündeln aus die Heber des Auges, von den hinteren medialen Bündeln der Rectus internus und vielleicht auch der Rectus inferior innervirt werden. Die pupillären Fasern des Oculomotorius hingegen würden beim Menschen in den vordersten Wurzelbündeln des Oculomotorius verlaufen. Ueber die Accommodation und die hierfür in Betracht kommenden Fasern wird nicht berichtet.

Westphal ${ }^{1}$ ) ist auf Grund der Untersuchung eines Failes von vollständiger Lähmung aller äusseren Augenmuskeln inclusive Levator palpebrae superior, bei dem auch die reflectorische Pupillarreaction fehlte, aber die Accommodation erhalten war, der Ansicht, dass die von ihm zuerst beschriebene kleinzellige Kerngruppe im vorderen Abschnitt des Oculomotoriuskernes der Innervation der interioren Muskeln vorstehe.

$\left.J_{a c o b}{ }^{2}\right)$ localisirt den Rectus superior und den Ciliarmuskel am weitesten nach vorn im Oeulomotoriushauptkern. Die Kerne für den Musculus ciliaris und Sphineter pupillae seien ränmlich beträchtlich getrennt.

In Kaiser's ${ }^{3}$ ) Fall waren nur die interioren Muskeln frei geblieben. Im Oeulomotoriuskern war der vorderste Theil des Oculomotoriuskernes "an der Seitenwand des dritten Ventrikels" versehont geblieben. Dahingegen war die "mediale und laterale Gruppe Westphal's" nicht unversehrt, sie könne also nicht der Kern der inneren Muskeln sein.

Auch v. Monakow ${ }^{4}$ ) ist der Ansicht, dass die EdingerWestphal'schen Zellgruppen als Ursprungszellen von Oculomotoriusfasern nieht aufzufassen sind. Er fand sie in einem Falle von vollständiger Ophthalmoplegie intaet, sämmtliche übrige Zellgruppen des Oculomotorius fehlten und der ganze Oculomotorius war degenerirt.

Eine ähnliche Beobachtung liegt auch von $B o ̈ t t i g e{ }^{5}$ ) vor.

Dahingegen fand v. Monakow ${ }^{4}$ ) in einem Falle von einseitiger Lähmung des Museulus sphineter und eiliaxis einen kleinen sklerotischen Heerd im lateralen Absebnitt des gleichseitigen vorderen Zweihügels. Der Arm des vorderen Zweihügels war vollständig degenerirt.

1) Loe. cit.

2) Deutsehe Zeitschrift f. Nervenheilk, V. S. 188. 1894.

9) Deutsche Zeitschrift f. Nervenheilk. VII.

4) Gehirnpathologie. Nothnagel's Specielle Pathologie und Therapie. IX. Bd. 1. Theil. Wien 1897.

5) Arch. f. Psychiatrie. Bd. XXI. Heft II. 1889. 
Ganz negative Resultate im Kerngebiet des Oculomotorius bezïglich der Kerne der Binnenmuskeln des Auges haben Schiff ${ }^{1}$ )Cassirer ${ }^{2}$ ), Stuelp ${ }^{3}$ ), Pineles ${ }^{4}$ ) n. A. erhalten.

In einem Falle, den v. Lenbe ${ }^{5}$ ) beschrieb, bestand bei einer Ptosis und Mydriasis eine Haemorrhagie im lateralen Theil des gleichseitigen Hauptkernes.

Spitzka ${ }^{\text {i) }}$ erwähnt eines nicht genauer publicirten Falles, wo linkseitige Ptosis durch einen haemorrhagischen Heerd, welcher "the most external sub-group of the main nest" zerstörte, hervorgerufen war.

Siemerling ${ }^{7}$ ) macht die Angabe, dass er in einem Falle von linksseitiger angeborener Ptosis in der Hauptgruppe des Oculomotoriuskernes beiderseits im proximalen Absehnitt degenerative Veränderungen gefunden habe.

Verfasser ${ }^{8}$ ) hatte selbst Gelegenheit, das Oculomotoriuskerngebiet eines Mädchens mit beiderseitiger congenitaler Ptosis zu untersuchen. Der Levator palpebrae superior war beiderseits schwach entwickelt. Im Kerngebiet konnte keine Abweichung: von den normalen Verhältnissen festgestellt werden (Weigertbehandlung). Da die Serie dureh das Kerngebiet des Oculomotorius and Trochlearis nicht ganz lückenlos war, ist jedoch diese Beobachtung nicht einwandsfrei.

Sachs ${ }^{9}$ ) fand bei einem zweijährigen Kinde nach einseitiger Exenteratio orbitae eine Degeneration in proximalen Theil des Kernes derselben und im distalen Abschnitt des Kernes der entgegengesetzten Seite. Der Edinger-Westphal'sche Kern derselben Seite soll ebenfalls verändert gewesen sein.

Es liegt noch eine Reihe weiterer pathologisch-anatomischer Untersuchungen vor. Einige Fälle sind klinisch oder mikroskopisch oder in beiderlei Hinsicht mangelhaft untersucht, in anderen Fällen wurden Veränderungen in Zellgruppen festgestellt, von denen wir jetzt wissen, dass

1) u. 2) Arbeiten aus Obersteiner's Laboratorium. 4. Heft.

3) v. Graefe's Arch. f. Ophthalm. XLI. 2.

4) Arbeiten aus Obersteiner's Laboratorium. 4. Heft.

b) Deutsches Arch. f. klin. Medicin. Bd. XL. S. 221.

6) The journ. of nerv. and ment. Dec. 1888.

7) Arch. f. Psychiatrie. Bd. XX́III. 3. Heft. 1892.

8) Centralbl. f. Nervenheilk. u. Psych. 1898. Febr.

3) v. Graefe's Arch. f. Ophthalm. Bd. XLII. 3. S. 40. 
Zur Lehre von den Augenmuskellähmungen ete. I. 385

sie gar nicht zum Oculomotoriuskern gehören - ein Eingehen auf diese Fälle erscheint nicht angebracht.

Aber anch sorgfältige Untersuchungen haben der angewandten Methoden halber nicht zu einwandfreien Ergebnissen führen könmen.

Wir wissen heutzutage, dass Fixirung mit Chromsalzen, Färbungen nach Weigert, mit Carmin, mit Nigrosin zum Studium der Veränderungen der Ganglienzellen sich nicht eignen, und dass die damit gewonnenen Resultate sehr mit Vorsicht aufzunehmen sind.

Wir wissen jetzt, dass auch unter normalen Verhältnissen alle möglichen Variationen in Bezug anf die Grösse der Zellen, das Verhalten der Fortsätze, das Verhalten Farbstoffen gegenüber etc. vorkommen.

Weiterhin ist bekannt und Verfasser hat Gelegenheit gehabt, sich auch hiervon zu überzeugen, dass alle möglichen im Körper gelegentlich vorhandenen Giftstoffe das Aussehen der Ganglienzellen ändern, dass schon wenige Stunden nach dem Tode bedeutende Veränderungen an den Ganglienzellen auftreten. Auch aus diesen Gründen kann und muss gegen die Beweiskraft vieler Befunde ein berechtigter Einwand erhoben werden.

Ich glaube, man kann mit gutem Recht behaupten, dass bisher die pathologisch-anatomische, speciell die mikroskopische Untersuchung fast keine absolut sicheren Resultate ergeben hat. Die Hoflnung ist jedoch berechtigt, dass die verbesserte Technik bald brauchbare und durchaus einwandfreie Resultate ergeben wird.

Erklärung der Abbildungen auf Taf. XIII-XVI, Fig. $1-14$.

Fig. 1-12 sind Frontalschnitte durch die Vierhügelgegend (Oculomotoriuskern). Genaue Angabe der Sehnittführung siehe S. 340. Vergrösserung Seibert. Obj. 0 , Oc. 0 .

Fig. 1-3 auf Taf. XIII sind Schnitte einer Weigert-Serie durch das Oculomotoriuskerngebiet des Kaninchens. Fig. 1 
386 L. Bach, Zur Lehre von den Augenmuskellähmungen etc. I.

(Schnitt 89) geht durch den distalsten Abschnitt des Oculomotoriuskernes, Fig. 2 (Schnitt 108) geht ungefähr durch die Mitte des Kernes. Fig. 3 (Schnitt 124) geht durch das proximale Drittel des Kerngebietes.

D. $L=$ Dorsales (hinteres) Längsbündel. G. $F=$ Bündel gekreuzter Fasern. Ug. $F .=$ Ungekreuzte Faserbündel. $G . C a . F .=$ Gekreuzte commissurenartig verlaufende Fasern. $K l . M$. $=$ Kleinzellige Mediankerne (Eding er Westphal'sche Kerne).

Fig. 4-6 auf Taf. XIII u. XIV sind Schnitte einer Weigert-Serie durch das Oculomotoriuskerngebiet einer nicht ganz ausgewachsenen Katze. Fig. 4 (Schnitt 36) geht durch den distalsten Bereich, Fig. 5 (Schnitt 72) ungefähr durch die Mitte, Fig. 6 (Schnitt 102) durch den proximalen Abschnitt des Kernes.

Bezeichnungen $=$ Fig. 1-3. - Auf Fig. 4 ist die oben an das Kerngebiet angrenzende Gehirnparthie eine Strecke weit mit abgebildet, um zu zeigen, dass daselbst sich dieselben Zellen befinden, wie in dem kleinzelligen Mediankeme.

Fig. 7-9 anf Taf. XTV n. XV sind Schnitte einer Weigert-Serie durch das Oculomotoriuskerngebiet eines kleinen Affen (Hapale Jacchus). Fig. 7 geht durch den distalsten Kernabschnitt, Fig. 8 ungefähr durch die Mitte, Fig. 9 durch das proximale Drittel des Kernes. Bei Fig. 9 sind die Hauptkerne nacb Schnitt 122, die kleinzelligen Kerne nach Schnitt 121 gezeichnet.

Bezeichnungen = Fig. 1-3.

Fig. 10-12 anf Taf. XV u. XVI sind Schnitte einer Weigert-Serie durch das Oculomotoriuskemgebiet eines erwachsenen Mannes. Fig. 10 (Schnitt 84 und 87) geht durch das distalste Kerngebiet, Fig. 11 (Schnitt 131 und 132) durch die vordere Hälfte des mittleren Drittels, Fig. 12 (Schnitt 160) durch den proximalsten Kernabschmitt.

$C . K=$ Perlia's Centralkern. W. $K l, D L . K=$ West phal's kleinzellige dorsolaterale Kerne. Oc. $H . K .=$ Oculomotoriushauptkern. Dar. $\boldsymbol{K}$. $=$ Darkschewitsch scher Kern. Die übrigen Bezeichnungen $=$ Fig. 1-3.

Fig. 13 und 14 auf Taf. XV sind Frontalschnitte durch verschiedene Abschnitte des Oculomotoriuskermes eines mensehlichen Embryo (ea. 34 Wochen) nach Golgi - Cajal behandelt, Sehnitt 6 und 26 der Serie. Vergrösserung Seibert Obj. I. Oc. 0. $\mathrm{Um} 1 / 8$ verkleinert. 

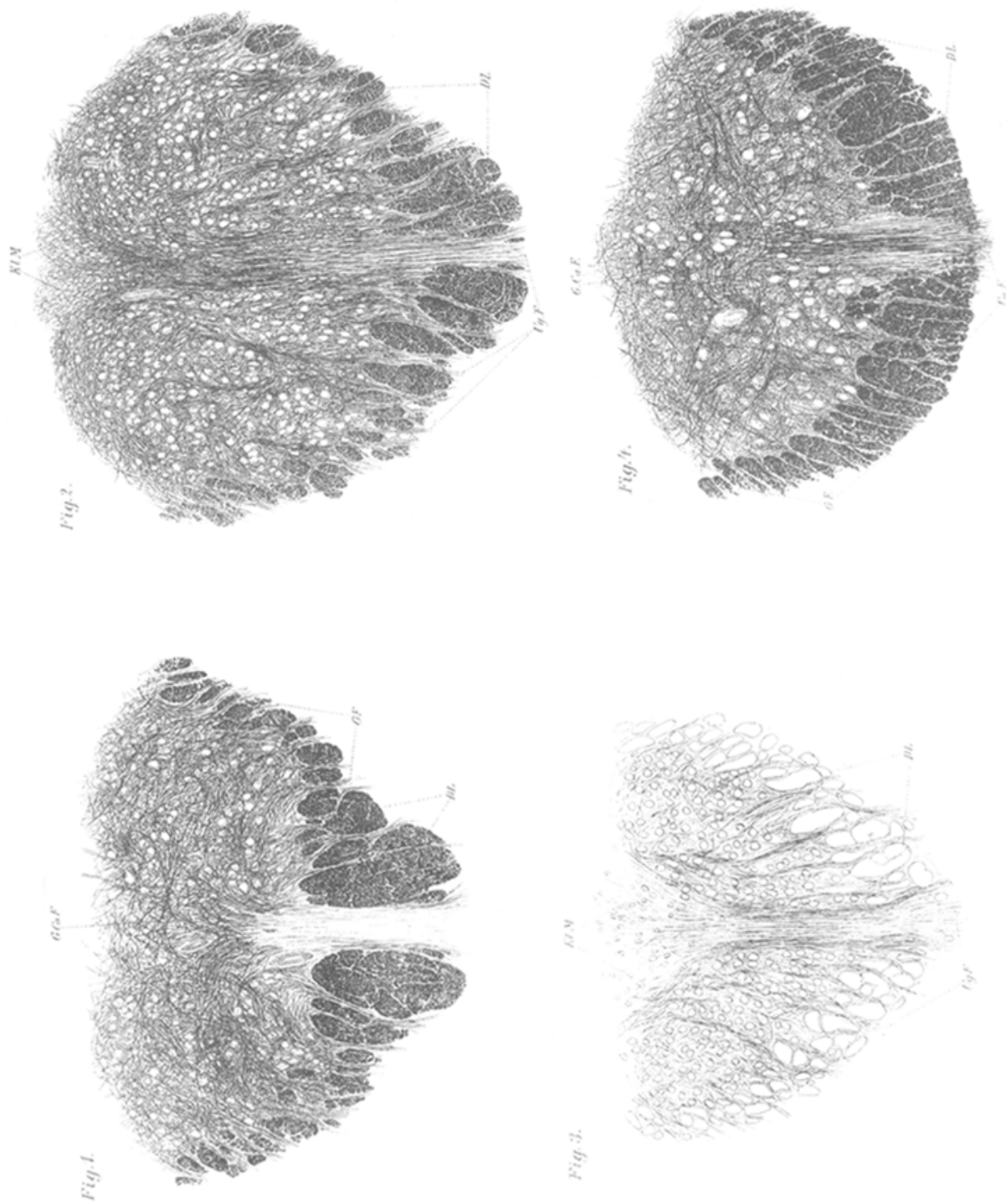

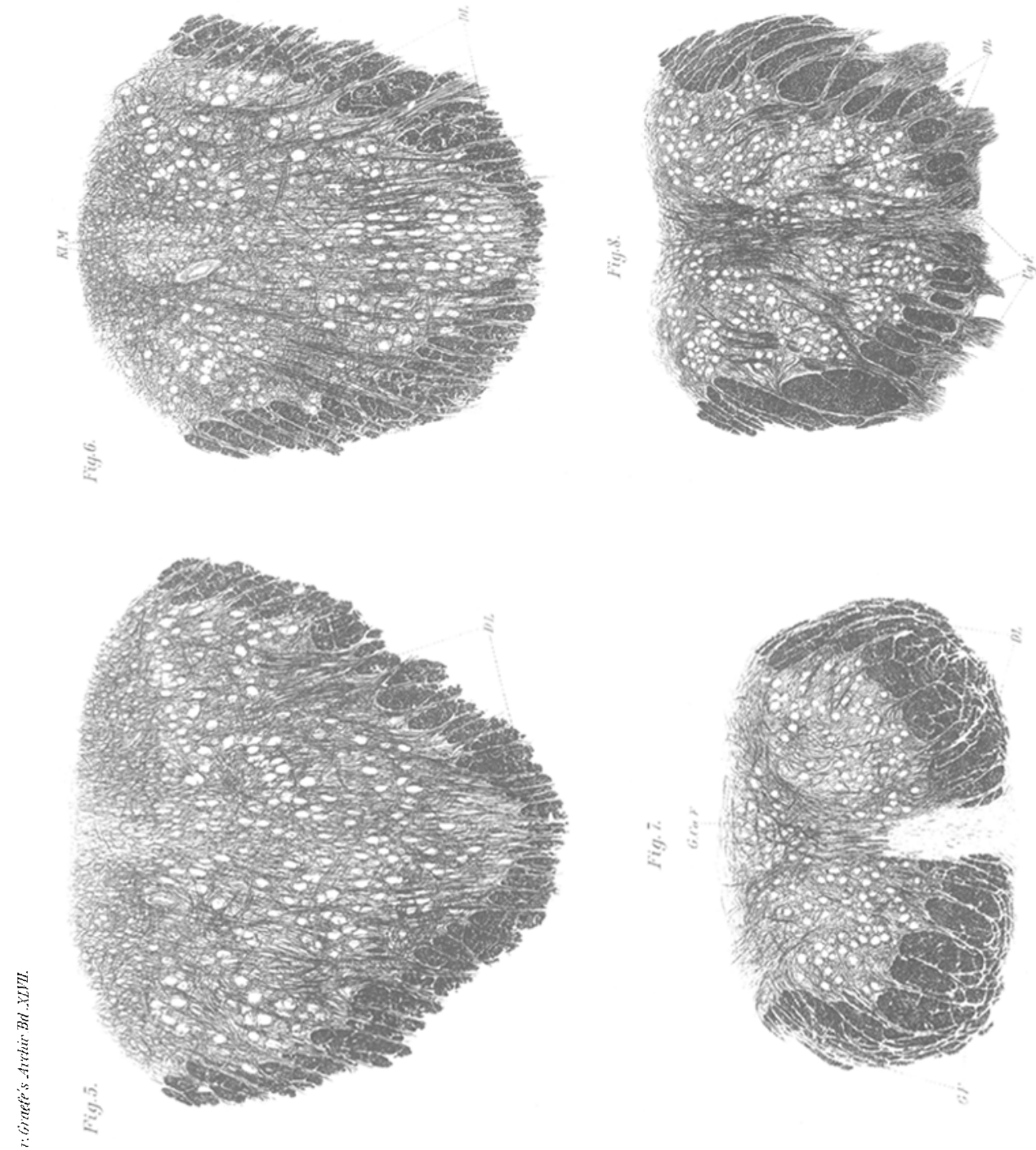

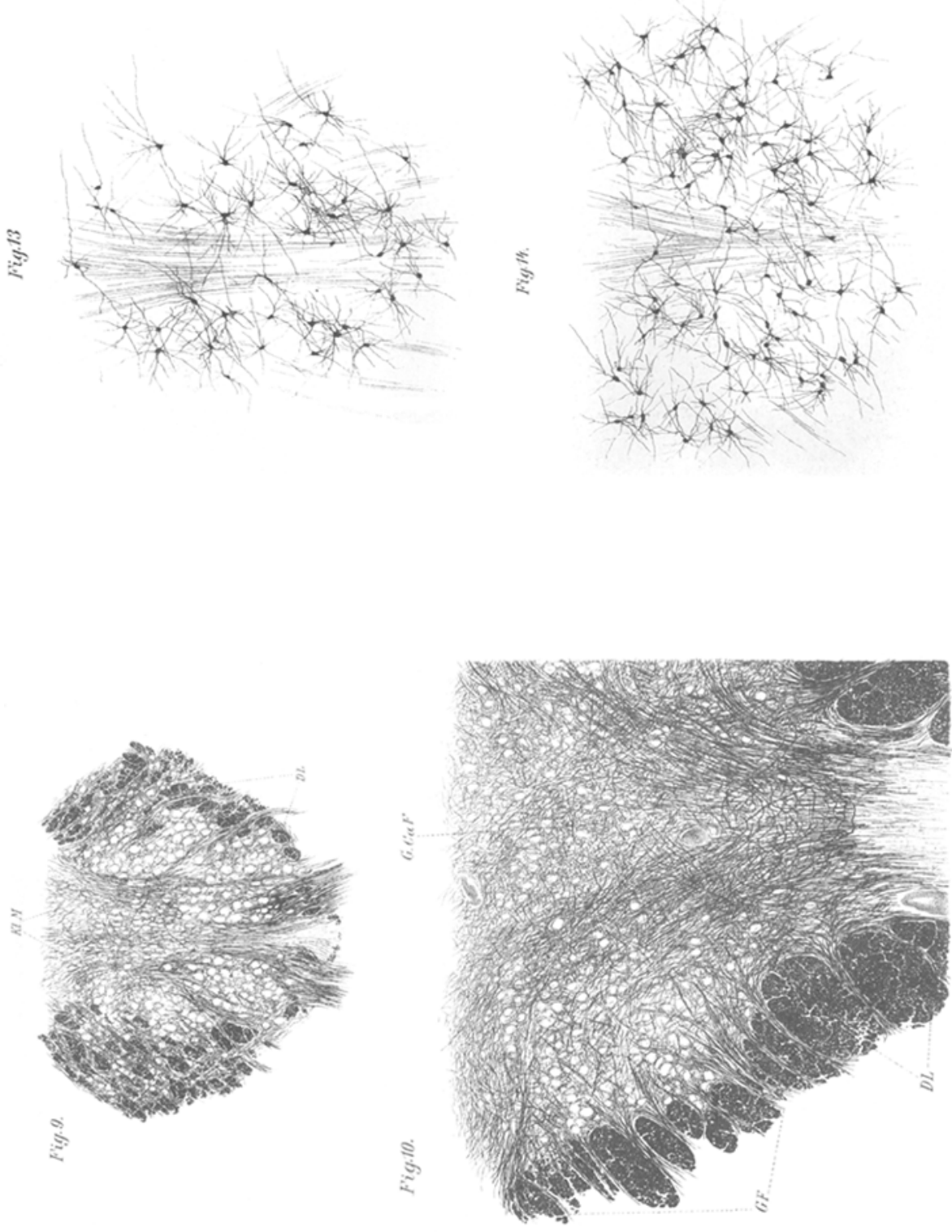


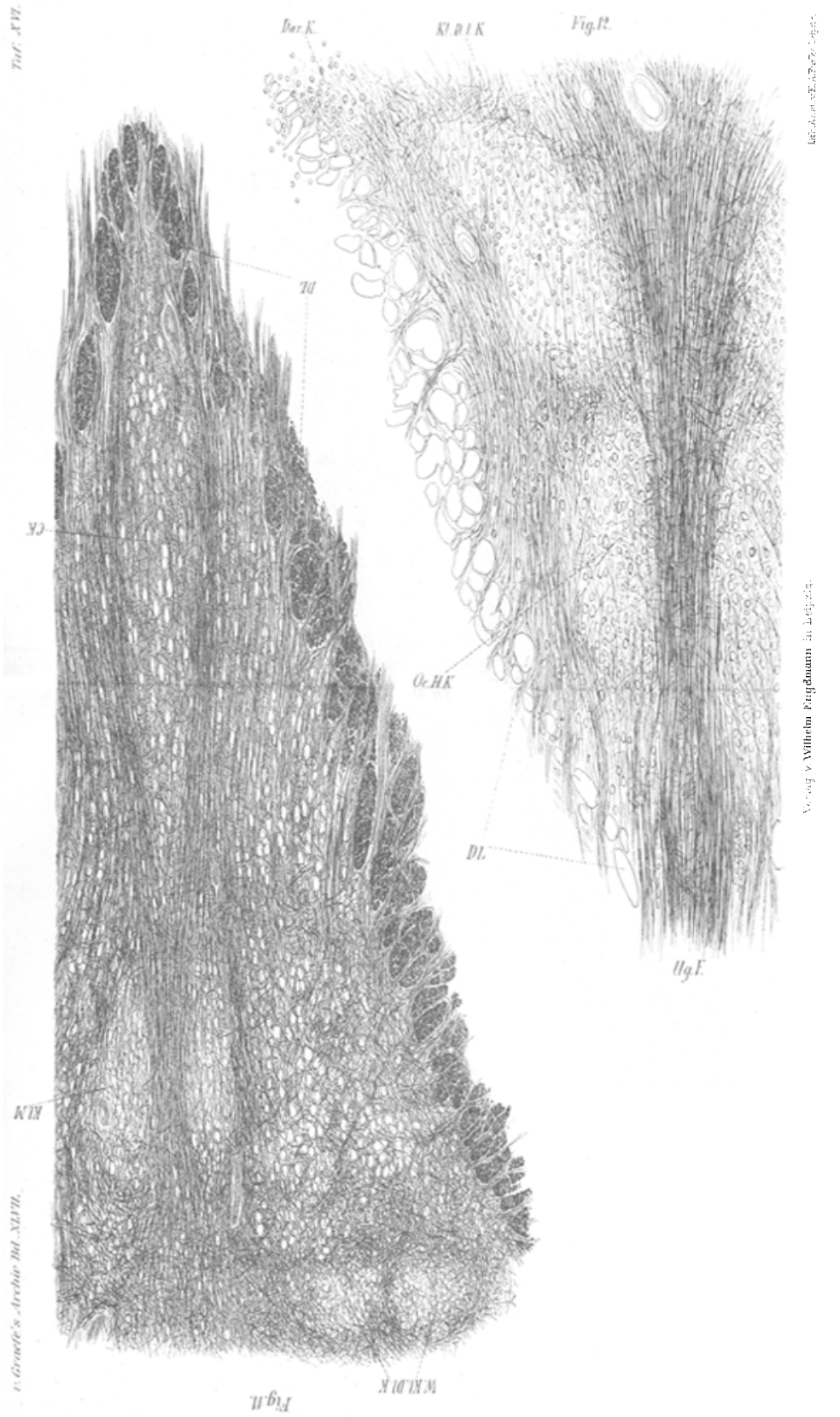

